\title{
A Divergence-Form Wave-Induced Pressure Inherent in the Extension of the Eliassen-Palm Theory to a Three-Dimensional Framework for All Waves at All Latitudes*
}

\author{
HIDENORI AIKI \\ Application Laboratory, Japan Agency for Marine-Earth Science and Technology, Yokohama, Japan \\ KOUTAROU TAKAYA \\ Department of Physics, Faculty of Science, Kyoto Sangyo University, Kyoto, Japan \\ RICHARD J. GREATBATCH \\ GEOMAR Helmholtz-Zentrum für Ozeanforschung Kiel, Kiel, Germany
}

(Manuscript received 17 June 2014, in final form 20 January 2015)

\begin{abstract}
Classical theory concerning the Eliassen-Palm relation is extended in this study to allow for a unified treatment of midlatitude inertia-gravity waves (MIGWs), midlatitude Rossby waves (MRWs), and equatorial waves (EQWs). A conservation equation for what the authors call the impulse-bolus (IB) pseudomomentum is useful, because it is applicable to ageostrophic waves, and the associated three-dimensional flux is parallel to the direction of the group velocity of MRWs. The equation has previously been derived in an isentropic coordinate system or a shallow-water model. The authors make an explicit comparison of prognostic equations for the IB pseudomomentum vector and the classical energy-based (CE) pseudomomentum vector, assuming inviscid linear waves in a sufficiently weak mean flow, to provide a basis for the former quantity to be used in an Eulerian time-mean (EM) framework. The authors investigate what makes the three-dimensional fluxes in the IB and CE pseudomomentum equations look in different directions. It is found that the two fluxes are linked by a gauge transformation, previously unmentioned, associated with a divergence-form wave-induced pressure $\Lambda$. The quantity $\Lambda$ vanishes for MIGWs and becomes nonzero for MRWs and EQWs, and it may be estimated using the virial theorem. Concerning the effect of waves on the mean flow, $\Lambda$ represents an additional effect in the pressure gradient term of both (the three-dimensional versions of) the transformed EM momentum equations and the merged form of the EM momentum equations, the latter of which is associated with the nonacceleration theorem.
\end{abstract}

\section{Introduction}

Generalization of classical wave-mean flow interaction theory concerning the Eliassen-Palm relation has attracted significant attention in the past decades (Eliassen and Palm 1960; Bühler 2014). The theory was originally developed in a zonal-mean framework for mountain waves and

\footnotetext{
* Supplemental information related to this paper is available at the Journals Online website: http://dx.doi.org/10.1175/JAS-D-14-0172.s1.

Corresponding author address: Hidenori Aiki, JAMSTEC, 3173-25, Showa-machi, Kanazawa-ku, Yokohama-city, Kanagawa 236-0001, Japan.

E-mail: aiki@jamstec.go.jp
}

reformulated by Andrews and McIntyre (1976, hereafter AM76) in the context of quasigeostrophic dynamics. Let $\overline{\bar{A}}$ and $A^{\dagger} \equiv A-\overline{\bar{A}}$ be the zonal mean (at fixed latitude and height) of an arbitrary quantity $A$ and the deviation from it, respectively. A zonal-mean quasigeostrophic expression for the Taylor-Bretherton identity (Taylor 1915; Bretherton 1966; Dritschel and McIntyre 2008) may be written as

$$
\begin{aligned}
\overline{\overline{v^{\dagger} Q^{\dagger}}} & =\partial_{y} F^{y}+\partial_{z} F^{z}, \\
F^{y} & \equiv \overline{\overline{\psi_{x} \psi_{y}}}=-\overline{\overline{\boldsymbol{v}^{\dagger} u^{\dagger}}}, \text { and } \\
F^{z} & \equiv-\left(\overline{\overline{\psi_{z} \psi_{x}}} / \overline{\bar{\rho}}_{z}\right) f_{0}^{2} \rho_{0} / g=\overline{\overline{\rho^{\dagger} \partial_{x} p^{\dagger}}} / \overline{\bar{\rho}}_{z}=f_{0} \overline{\overline{\rho^{\dagger} \boldsymbol{v}^{\dagger}}} / \overline{\bar{\rho}}_{z},
\end{aligned}
$$

DOI: 10.1175/JAS-D-14-0172.1 
TABLE 1 . List of symbols, where $A$ is an arbitrary quantity.

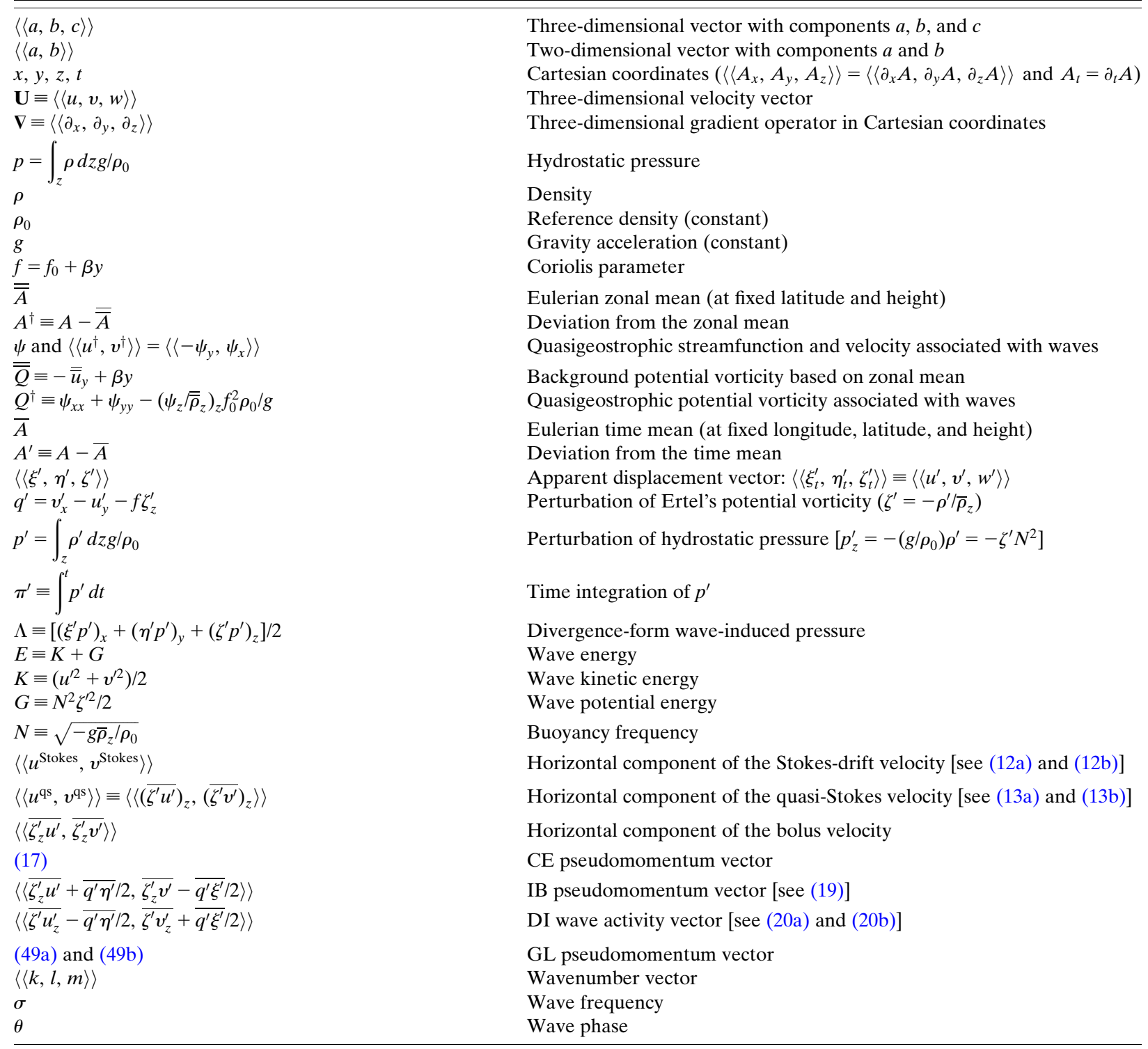

where $Q^{\dagger}=\psi_{x x}+\psi_{y y}-\left(\psi_{z} / \overline{\bar{\rho}}_{z}\right)_{z} f_{0}^{2} \rho_{0} / g$ is quasigeostrophic potential vorticity associated with waves; $p^{\dagger}=\psi f_{0}$ and $\rho^{\dagger}=\psi_{z}\left(-f_{0} \rho_{0} / g\right)$ are the perturbations of hydrostatic pressure and density, respectively; and $\left\langle\left\langle F^{y}, F^{z}\right\rangle\right\rangle$ is referred to as the (quasigeostrophic) EliassenPalm flux. The Cartesian coordinate system is represented by independent variables $x, y, z$, and $t$, where $x, y$, and $z$ each increase eastward, northward, and vertically upward, respectively, with the corresponding three-dimensional components of velocity being written as $\langle\langle u, v, w\rangle\rangle$ and the Boussinesq approximation has been used. The other symbols are mostly conventional and explained in both Table 1 and section 2. Combining $\left(\partial_{t}+\overline{\bar{u}} \partial_{x}\right) Q^{\dagger}+v^{\dagger} \partial_{y} \overline{\bar{Q}}=0$ with (1a) yields a zonal-mean quasigeostrophic expression for the Eliassen-Palm relation (Bretherton 1966; AM76):

$$
\partial_{t}\left[\overline{\overline{Q^{\dagger 2}}} /\left(2 \overline{\bar{Q}}_{y}\right)\right]+\partial_{y} F^{y}+\partial_{z} F^{z}=0,
$$

which represents a conservation equation for (the linear wave version of) the quasigeostrophic wave activity. In the present paper, the term "wave activity" is always associated with the variance of vorticity perturbations, to be explained later. Noting that $\overline{\overline{\partial_{x} p}}=0$ in a zonally periodic domain, a standard Eulerian zonal-mean equation for the zonal component of velocity is written as $\partial_{t} \overline{\bar{u}}-f_{0} \overline{\bar{v}}_{a}=-\partial_{y}\left(\overline{\overline{v^{\dagger} u^{\dagger}}}\right)$, where $\overline{\bar{v}}_{a}$ is the meridional 
component of the ageostrophic Eulerian zonal-mean velocity. AM76 have suggested rewriting this equation into an expression, which may be interpreted in terms of the wave dynamics:

$$
\partial_{t} \overline{\bar{u}}-f_{0} v^{*}=\partial_{y} F^{y}+\partial_{z} F^{z}
$$

which is referred to as the transformed Eulerian-mean (TEM) momentum equation, and $v^{*} \equiv \overline{\bar{v}}_{a}+\left(-\overline{\overline{\rho^{\dagger} \boldsymbol{v}^{\dagger}} / \overline{\bar{\rho}}_{z}}\right)_{z}$ is the meridional component of the sum of the ageostrophic Eulerianl zonal-mean velocity and the waveinduced velocity. Substitution of (2) into the TEM momentum equation (3) yields

$$
\partial_{t}\left[\overline{\bar{u}}+\overline{\overline{Q^{\dagger 2}}} /\left(2 \overline{\bar{Q}}_{y}\right)\right]-f_{0} v^{*}=0
$$

which is closely related with the nonacceleration theorem of Charney and Drazin (1961). Equation (4) is an example of what is referred to as the "merged" form of the Eulerian-mean (MEM) momentum equation in the present study.

The important properties of the Eliassen-Palm flux, in the context of a zonal-mean framework, have been summarized in section 1 of Plumb (1986, hereafter P86), as follows:

1) For small-amplitude waves on a zonal flow, $\left\langle\left\langle F^{y}, F^{z}\right\rangle\right\rangle$ appears as the flux of wave activity in a conservation relation, relating $\partial_{y} F^{y}+\partial_{z} F^{z}$ to wave transience and nonconservative effects, as illustrated for wave transience in (2) of the present paper.

2) In the Wentzel-Kramers-Brillouin (WKB) limit of almost-plane waves on a slowly varying mean flow, $\left\langle\left\langle F^{y}, F^{z}\right\rangle\right\rangle$ is parallel to the group velocity (for Rossby waves).

3) For quasigeostrophic flow, $\partial_{y} F^{y}+\partial_{z} F^{z}$ is proportional to the northward eddy flux of quasigeostrophic potential vorticity, as shown by (1a) of the present paper.

4) The quasigeostrophic momentum and thermodynamic equations may be transformed in such a way that the only term describing eddy-mean flow interaction is an effective zonal force, proportional to $-\left(\partial_{y} F^{y}+\partial_{z} F^{z}\right)$; thus, $\left\langle\left\langle F^{y}, F^{z}\right\rangle\right\rangle$ may be regarded as an effective flux of easterly momentum, as shown by (3) of the present paper.

Various attempts have been made to extend the above framework to (i) a three-dimensional framework for diagnosing the interaction between waves and the mean flow in the horizontal plane and (ii) ageostrophic waves in both midlatitude and equatorial regions (Andrews and McIntyre 1978a, hereafter
AM78a; Ripa 1982; Hoskins et al. 1983; Held 1985; Trenberth 1986; Haynes 1988; Scinocca and Shepherd 1992, hereafter SS92; Sassi and Garcia 1997; Sato and Dunkerton 1997; Greatbatch 1998, 2001; Horinouchi and Yoden 1998). On the other hand, an equivalent for the time-mean and nonlinear version of the MEM momentum equation (4) has been widely used in the community of oceanic surface gravity waves as a basis for ocean circulation models to include the effects of both the Craik and Leibovich (1976) vortex force and also the transfer of momentum from waves to circulation associated with the dissipation of wave energy (Ardhuin et al. 2008; Aiki and Greatbatch 2014, hereafter AG14).

The present study is aimed at unifying the above framework to all midlatitude inertia-gravity waves (MIGWs), midlatitude Rossby waves (MRWs), and equatorial waves (EQWs; including equatorial inertiagravity, Kelvin, mixed Rossby-gravity, and Rossby waves) in the atmosphere and ocean (Matsuno 1966; Yanai and Maruyama 1966; Gill 1982; Holton 1992). To our knowledge, the concept of wave activity (or pseudomomentum) has at least five kinds of variants:

(i) Wave activity based on the variance of Ertel's potential vorticity (EPV), which has been used in studies of quasigeostrophic Rossby waves (AM76, P86), as explained above

(ii) Wave activity based on the variance of relative vorticity associated with wave motions in the vertical plane that has been used mainly in the studies of nonhydrostatic gravity waves (SS92)

(iii) The so-called bolus velocity, which has been used mainly in the studies of hydrostatic waves in either an isentropic coordinate system or a shallow-water model (Rhines 1982)

(iv) Wave energy divided by the apparent phase speed of waves that has been used mainly in studies of both gravity and planetary wave literatures (Whitham 1974)

(v) The generalized Lagrangian pseudomomentum of AM78a that has been used mainly in studies of both hydrostatic and nonhydrostatic gravity waves (Bühler 2009),

where linear waves are assumed for simplicity [i.e., finite-amplitude wave versions of the quantities (i) and (ii) are written in the forms of the integral (rather than the variance) of vorticity]. Takaya and Nakamura (1997, 2001, hereafter TN01) have used the combination of the quantities (i) and (iv) to consider the wave activity of stationary MRWs in the context of a three-dimensional framework. The quantity (iv) is fundamental but requires a dispersion relation for each type of wave; thus, it is not suitable for a unified treatment of MIGWs, MRWs, and 
EQWs. The quantity (v) has not been widely used in previous studies for planetary waves (the planetary $\beta$ effect is implicit in the original paper by AM78a). Solomon and Nakamura (2012) have suggested using a gauge transformation to understand the difference between the quantities (i) and (v). On the other hand, Ripa (1982) and Andrews (1983a) have suggested that the combination of the quantities (i) and (iii) may be used as a wave activity associated with ageostrophic waves. This approach has led to a three-dimensional and ageostrophic version of the Eliassen-Palm relation [shown in (2)] that has been derived by Haynes (1988) using an isentropic coordinate system. However, it has been a challenge in atmospheric science to extend the basic result of Ripa (1982), Andrews (1983a), and Haynes (1988) (specialized to a shallow-water model or an isentropic coordinate system) to obtain the three-dimensional and ageostrophic version of the TEM and MEM momentum equations.

Given the cost to handle all MIGWs, MRWs, and EQWs, the present study adopts an Eulerian time-mean framework for small-amplitude waves in a hydrostatic Boussinesq fluid with a sufficiently weak mean flow. The present paper is organized as follows. In section 2, we explain what we call the impulse-bolus pseudomomentum vector and its relationship to the classical energy-based pseudomomentum vector. In section 3 , by comparing prognostic equations for the impulse-bolus and classical energy-based pseudomomenta, we investigate what makes the impulse-bolus and classical energy-based fluxes look in different directions. It is found that the two fluxes are linked by a gauge transformation, previously unmentioned, associated with a divergence-form waveinduced pressure $\Lambda$. We investigate the characteristics of $\Lambda$ depending on MIGWs, MRWs, and EQWs. In section 4, we show that the quantity $\Lambda$ is a cornerstone for obtaining the three-dimensional and ageostrophic versions of the TEM and MEM momentum equations [see (3) and (4)]. In section 5, we explain how $\Lambda$ has been hidden in previous formulations for the wave-mean flow interaction. Section 6 presents a brief summary.

\section{Mathematical development}

We consider small-amplitude wave motions in a continuously stratified fluid in a rotating frame. In the rest of the present paper, we use a low-pass temporal filter to decompose an arbitrary quantity $A$ into the mean and perturbation components: $A=\bar{A}+A^{\prime}$, where the overbar and prime indicate the Eulerian time-mean (EM) at fixed longitude, latitude, and height and the deviation from it, respectively (not to be confused with $\overline{\bar{A}}$ and $A^{\dagger}$; see Table 1). The details of the derivations of some equations are shown in the supplemental material.

\section{a. Standard EM momentum equations}

The EM momentum equations for a hydrostatic, Boussinesq, inviscid fluid in a rotating frame are given by

$$
\begin{gathered}
\bar{u}_{t}+\nabla \cdot(\overline{\mathbf{U}} \bar{u})-f \bar{v}=-\bar{p}_{x}-\nabla \cdot\left\langle\left\langle\overline{u^{\prime} u^{\prime}}, \overline{v^{\prime} u^{\prime}}, \overline{w^{\prime} u^{\prime}}\right\rangle\right\rangle, \\
\bar{v}_{t}+\nabla \cdot(\overline{\mathbf{U}} \bar{v})+f \bar{u}=-\bar{p}_{y}-\nabla \cdot\left\langle\left\langle\overline{u^{\prime} v^{\prime}}, \overline{v^{\prime} v^{\prime}}, \overline{w^{\prime} v^{\prime}}\right\rangle\right\rangle, \\
0=-\bar{p}_{z}-\left(g / \rho_{0}\right) \bar{\rho}
\end{gathered}
$$

where $f=f_{0}+\beta y$ is the Coriolis parameter; $p$ is hydrostatic pressure divided by the reference density $\rho_{0}$ of air (or seawater), with $g$ being the acceleration due to gravity; $\boldsymbol{\nabla} \equiv\left\langle\left\langle\partial_{x}, \partial_{y}, \partial_{z}\right\rangle\right\rangle$; and $\mathbf{U} \equiv\langle\langle u, v, w\rangle\rangle$. In (5a) and $(5 b)$ the effect of waves on the mean flow has been represented by the divergence of the three-dimensional Reynolds stress. Throughout the present study (i) the spatial scale for variations of the mean flow is assumed to be sufficiently larger than the wavelength for the WKB approximation to be valid; (ii) the mean flow is assumed to be sufficiently weak, ${ }^{1}$ as explained below; and (iii) $\rho_{0}$ is assumed to be a three-dimensionally uniform constant (as in oceanic studies). The assumptions (ii) and (iii) are for simplicity, given the cost to achieve a unified treatment of MIGWs, MRWs, and EQWs.

\section{b. Governing equations for linear hydrostatic waves}

Letting $\epsilon \ll 1$ be a small nondimensional parameter representing the amplitude of the waves, the present study assumes that the magnitude of the velocity of both the mean flow and the wave motions is one order, in terms of $\epsilon$, smaller than the magnitude of the phase speed of the waves. Governing equations for linear waves in a rotating stratified fluid may be written using the Boussinesq, hydrostatic, and inviscid approximations:

$$
\begin{aligned}
u_{t}^{\prime}-f v^{\prime} & =-p_{x}^{\prime}, \\
v_{t}^{\prime}+f u^{\prime} & =-p_{y}^{\prime}, \\
\rho_{t}^{\prime}+w^{\prime} \bar{\rho}_{z} & =0, \\
p^{\prime} & =g \int_{z} \rho^{\prime} d z / \rho_{0}, \quad \text { and } \\
u_{x}^{\prime}+v_{y}^{\prime}+w_{z}^{\prime} & =0,
\end{aligned}
$$

where the effect of the mean flow does not appear because of the scaling mentioned above. Equations

\footnotetext{
${ }^{1}$ All the results of the present study are invariant under the Galilean transformation associated with a uniform nonweak mean flow in the zonal direction (not shown).
} 
TABLE 2. Characteristics of midlatitude and equatorial waves. The third column $\left(A_{y y}^{\prime} \simeq-l^{2} A^{\prime}\right)$ indicates whether waves are nearly plane in the meridional direction, where $A^{\prime}$ is an arbitrary quantity and $l$ is the wavenumber in the meridional direction. Symbols in the last three columns are defined by $q^{\prime} \equiv v_{x}^{\prime}-u_{y}^{\prime}-f \zeta_{z}^{\prime}, \Lambda \equiv\left[\left(\xi^{\prime} p^{\prime}\right)_{x}+\left(\eta^{\prime} p^{\prime}\right)_{y}+\left(\zeta^{\prime} p^{\prime}\right)_{z}\right] / 2, K \equiv\left(u^{\prime 2}+v^{\prime 2}\right) / 2, G \equiv\left(N^{2} / 2\right) \zeta^{\prime 2}$, and $E \equiv K+G$.

\begin{tabular}{|c|c|c|c|c|c|}
\hline Type of waves & Acronym & $A_{y y}^{\prime} \simeq-l^{2} A^{\prime}$ & $\eta^{\prime}=-q^{\prime} / \beta$ & $\bar{\Lambda}$ & $\left(\overline{v^{\prime} \xi^{\prime}-u^{\prime} \eta^{\prime}}\right) / 2$ \\
\hline Midlatitude inertia-gravity waves & MIGWs & Yes & No & (37) and 0 & (B3) and $(\bar{K}-\bar{G}) / f$ \\
\hline Midlatitude Rossby waves & MRWs & Yes & Yes & (36), (37), and $-\bar{E}$ & (B2), (B3), and $2 \bar{K} / f$ \\
\hline Equatorial Rossby waves & EQWs & No & Yes & (36) and (37) & (B2) and (B3) \\
\hline Equatorial mixed Rossby-gravity waves & EQWs & No & Yes & (36) and (37) & (B2) and (B3) \\
\hline Equatorial Kelvin waves & EQWs & No & Yes & (36) and (37) & (B2) and (B3) \\
\hline Equatorial inertia-gravity waves & EQWs & No & Yes & (36) and (37) & (B2) and (B3) \\
\hline
\end{tabular}

(6a)-(6e) are applicable to all MIGWs, MRWs, and EQWs (Gill 1982; Holton 1992). For convenience, we introduce an apparent displacement vector $\left\langle\left\langle\xi^{\prime}, \eta^{\prime}, \zeta^{\prime}\right\rangle\right\rangle$ associated with the perturbation velocity:

$$
\left\langle\left\langle u^{\prime}, v^{\prime}, w^{\prime}\right\rangle\right\rangle=\left\langle\left\langle\xi_{t}^{\prime}, \eta_{t}^{\prime}, \zeta_{t}^{\prime}\right\rangle\right\rangle,
$$

where $0=\overline{\xi^{\prime}}=\overline{\eta^{\prime}}=\overline{\zeta^{\prime}}$ should be understood. The incompressible condition (6e) may be rewritten as

$$
\xi_{x}^{\prime}+\eta_{y}^{\prime}+\zeta_{z}^{\prime}=0
$$

On the other hand, time integration of (6c) yields

$$
\zeta^{\prime}=-\rho^{\prime} / \bar{\rho}_{z}=\left(g / \rho_{0}\right) \rho^{\prime} / N^{2}=-p_{z}^{\prime} / N^{2}
$$

where (6d) and (7) have been used, and $N \equiv \sqrt{-g \bar{\rho}_{z} / \rho_{0}}$ is the buoyancy frequency, which is assumed to be uniform in the horizontal direction.

Taking the horizontal curl of (6a) and (6b) yields an equation for the development of the perturbation of $\mathrm{EPV}\left(q^{\prime} \equiv v_{x}^{\prime}-u_{y}^{\prime}-f \zeta_{z}^{\prime}\right)^{2}$

$$
q_{t}^{\prime}+\beta v^{\prime}=0,
$$

where (6e) has been used. Time integration of (10) yields

$$
q^{\prime}+\beta \eta^{\prime}=0
$$

where (7) has been used. Equations (10) and (11) are applicable to all MIGWs, MRWs, and EQWs, with the understanding that $q^{\prime}=0$ (i.e., $\beta=0$ ) and $v^{\prime} \neq 0$ for MIGWs, and $q^{\prime}=0$ (i.e., $v^{\prime}=0$ ) and $\beta \neq 0$ for equatorial Kelvin waves (Gill 1982; Müller 1995). Hence, $\eta^{\prime}=-q^{\prime} / \beta$ is valid for both MRWs and EQWs but should not be used for MIGWs (Table 2).

\footnotetext{
${ }^{2}$ The quantity $\zeta_{z}^{\prime}$ corresponds to (an Eulerian approximation for) the perturbation of nondimensionalized thickness in shallowwater equations. EPV may be approximated as $\left(v_{x}^{\prime}-u_{y}^{\prime}+\bar{v}_{x}-\right.$ $\left.\bar{u}_{y}+f\right) /\left(1+\zeta_{z}^{\prime}\right) \simeq\left(v_{x}^{\prime}-u_{y}^{\prime}+\bar{v}_{x}-\bar{u}_{y}+f\right)\left(1-\zeta_{z}^{\prime}\right)=\left(\bar{v}_{x}-\bar{u}_{y}+f\right)+$ $v_{x}^{\prime}-u_{y}^{\prime}-\left(\bar{v}_{x}-\bar{u}_{y}+f\right) \zeta_{z}^{\prime}$, the perturbation component of which reduces to $v_{x}^{\prime}-u_{y}^{\prime}-f \zeta_{z}^{\prime} \equiv q^{\prime}$ under the WKB approximation.
}

\section{c. The Stokes-drift velocity and the quasi-Stokes velocity}

The Stokes-drift velocity is defined as the difference between the Lagrangian-mean (LM) velocity and the EM velocity. An Eulerian approximation for the Stokesdrift velocity may be written as

$$
\begin{aligned}
u^{\text {Stokes }} & \equiv \overline{\xi^{\prime} u_{x}^{\prime}+\eta^{\prime} u_{y}^{\prime}+\zeta^{\prime} u_{z}^{\prime}} \\
& =\left(\overline{\xi^{\prime} u^{\prime}}\right)_{x}+\left(\overline{\eta^{\prime} u^{\prime}}\right)_{y}+\left(\overline{\zeta^{\prime} u^{\prime}}\right)_{z} \quad \text { and } \\
v^{\text {Stokes }} & \equiv \overline{\xi^{\prime} v_{x}^{\prime}+\eta^{\prime} v_{y}^{\prime}+\zeta^{\prime} v_{z}^{\prime}} \\
& =\left(\overline{\xi^{\prime} v^{\prime}}\right)_{x}+\left(\overline{\eta^{\prime} v^{\prime}}\right)_{y}+\left(\overline{\zeta^{\prime} v^{\prime}}\right)_{z},
\end{aligned}
$$

where a Taylor expansion in the three-dimensional direction has been used and, as throughout this paper, $\epsilon \ll 1$ (Longuet-Higgins 1953). Equations (12a) and (12b) exclude the effect of the shear of the mean flow under the assumption of a sufficiently weak mean flow in the present study.

On the other hand, the wave-induced velocity in the TEM theory of AM76 may be generalized to an Eulerian time-mean framework:

$$
\begin{aligned}
& u^{\mathrm{qs}} \equiv\left(\overline{\zeta^{\prime} u^{\prime}}\right)_{z}=\left(-\overline{\rho^{\prime} u^{\prime}} / \bar{\rho}_{z}\right)_{z} \quad \text { and } \\
& v^{\mathrm{qs}} \equiv\left(\overline{\zeta^{\prime} \boldsymbol{v}^{\prime}}\right)_{z}=\left(-\overline{\rho^{\prime} \boldsymbol{v}^{\prime}} / \bar{\rho}_{z}\right)_{z} .
\end{aligned}
$$

The mathematical expression in (13a) and (13b) is widely known in the atmospheric literature. ${ }^{3}$ However, we could not find an iconic name for this velocity that may be comparable to the Stokes-drift velocity. Therefore, the

\footnotetext{
${ }^{3}$ AM76 have used the quasi-Stokes velocity rather than the Stokes-drift velocity. Probably, this is because (i) both $\left(\overline{\xi^{\prime} v^{\prime}}\right)_{x}$ and $\left(\overline{\eta^{\prime} v^{\prime}}\right)_{y}$ in (12b) vanish in a zonal-mean framework for "neutral" waves and (ii) AM76 have also considered a residual-mean formulation for tracer equations in which the quasi-Stokes velocity naturally appears. Some later studies have developed advanced forms of residual-mean tracer equations that contain (variants of) the Stokes-drift velocity or the asymmetric component of the generalized diffusion tensor (Eden et al. 2007; Noda 2010).
} 
present paper uses the term "the quasi-Stokes velocity" to refer to the wave-induced velocity in (13a) and (13b). Indeed, under the approximations adopted in the present study (i.e., sufficiently weak mean flows and small-amplitude linear waves), the vertical derivative of the quasi-Stokes streamfunction in (4b) of McDougall and McIntosh (2001) reduces to (13a) and (13b). A generalized expression for the quasi-Stokes streamfunction is $\left\langle\left\langle\overline{\int_{z}^{z+\zeta^{\prime}} u d z}, \overline{\int_{z}^{z+\zeta^{\prime}} v d z}\right\rangle\right\rangle$, which may be traced back to (12) of Hasselmann (1971) and Fig. 2 of Longuet-Higgins (1969).

\section{d. Energy equations}

Wave energy is written as $E \equiv K+G$, where $K \equiv$ $\left(u^{\prime 2}+v^{\prime 2}\right) / 2$ is the wave kinetic energy and $G \equiv\left(N^{2} / 2\right) \zeta^{\prime 2}$ is the wave potential energy (Table 1 ). Multiplying (6a), (6b), and (9) by $u^{\prime}, v^{\prime}$, and $N^{2} w^{\prime}$, respectively, and then taking the sum of the three equations yields a conservation equation for $E$ :

$$
E_{t}+\nabla \cdot\left\langle\left\langle u^{\prime} p^{\prime}, v^{\prime} p^{\prime}, w^{\prime} p^{\prime}\right\rangle\right\rangle=0,
$$

which has been written as an instantaneous expression. ${ }^{4}$ It is known that the three-dimensional pressure flux $\left\langle\left\langle u^{\prime} p^{\prime}, v^{\prime} p^{\prime}, w^{\prime} p^{\prime}\right\rangle\right\rangle$ in (14) looks, after application of a low-pass time filter, in the direction of the group velocity of MIGWs but not of MRWs (Longuet-Higgins 1964; Masuda 1978; Durran 1988; Chang and Orlanski 1994; Cai and Huang 2013). Using (7) and (9), we derive another expression for the wave energy:

$E \equiv\left(u^{\prime 2}+v^{\prime 2}+N^{2} \zeta^{\prime 2}\right) / 2=\left(u^{\prime} \xi_{t}^{\prime}+v^{\prime} \eta_{t}^{\prime}-\zeta^{\prime} \pi_{z t}^{\prime}\right) / 2$,

where $\pi^{\prime} \equiv \int^{t} p^{\prime} d t$. The quantity $\pi^{\prime}$ is related to both the velocity and the displacement as

$$
\begin{aligned}
& u^{\prime}-f \eta^{\prime}=-\pi_{x}^{\prime} \text { and } \\
& v^{\prime}+f \xi^{\prime}=-\pi_{y}^{\prime},
\end{aligned}
$$

\footnotetext{
${ }^{4}$ Key equations have been derived as instantaneous expressions in the present study, following Andrews (1983b), Plumb (1985), and SS92. There are at least three separate approaches to represent the slow variations (in both the time space and the threedimensional space) of waves and mean flows (i.e., the WKB approximation). The first approach is to use an instantaneous expression when writing wave energy and pseudomomentum equations, as shown in Andrews (1983b), Plumb (1985), SS92, and the present study. The second approach is to use the set of the wave action and crest equations, which assumes wave amplitude, wavenumber, and wave frequency all have slow variations (Bretherton and Garrett 1968; Uryu 1974; Whitham 1974). The third approach is to systematically decompose an equation system for waves based an asymptotic expansion (cf. Chu and Mei 1970; Aiki and Greatbatch 2013). Both the second and third approaches require, at least, a dispersion relation for the given type of waves to be derived. Hence, the present study adopts the first approach to achieve a unified treatment of the different types of waves.
}

which have been derived by taking the time integral of (6a) and (6b). Equations (15)-(16b) prove useful in the next subsection.

\section{e. Two types of pseudomomentum vector}

In the classical linear wave theory (Bretherton and Garrett 1968; Uryu 1974; Whitham 1974), the pseudomomentum is defined as the vector with components given by the phase average of $E$ divided by $\sigma / k$ and $\sigma / l$, respectively (where $\sigma$ is the wave frequency and $\langle\langle k, l\rangle\rangle$ is the horizontal wavenumber vector), that is hereafter referred to as the classical energy-based (CE) pseudomomentum. In the present study, we assume a transient planar waveform so that a phase average is also a time average. Thus, the phase average of $E$ is interpreted as $\bar{E}$ : namely, a low-pass time-filtered wave energy. On the other hand, for $\epsilon \ll 1$, basic quantities associated with MIGWs and MRWs may be written in the form $\mathcal{A} F(z) \exp [i(k x+l y-\sigma t)]$, while quantities associated with EQWs may be written in the form $\mathcal{A} F(y, z) \exp [i(k x-\sigma t)]$. Both forms allow for slow variations in wave amplitude $\mathcal{A}$, the horizontal wavenumbers $k$ and $l$, wave frequency $\sigma$, and a vertically varying $N$. It can be said that both MIGWs and MRWs are nearly plane in the horizontal direction, while EQWs are nonplane in the meridional direction (Table 2). It is rather difficult to define the meridional wavenumber $l$ for EQWs. Furthermore, if the use of the wave action and crest equations (to derive prognostic equations for the pseudomomentum) is considered, it is laborious to derive dispersion relations for all the types of waves of interest.

To avoid the above problems, we introduce a generalized expression ${ }^{5}$ for the $\mathrm{CE}$ pseudomomentum vector by replacing the subscript $t$ in (15) with $x$ and $y$ and then applying a low-pass temporal filter, as follows:

$$
\left\langle\left\langle-\left(\overline{u^{\prime} \xi_{x}^{\prime}+v^{\prime} \eta_{x}^{\prime}-\zeta^{\prime} \pi_{z x}^{\prime}}\right) / 2,-\left(\overline{u^{\prime} \xi_{y}^{\prime}+v^{\prime} \eta_{y}^{\prime}-\zeta^{\prime} \pi_{z y}^{\prime}}\right) / 2\right\rangle\right\rangle,
$$

which does not explicitly contain the wavenumber, the wave frequency, or the phase speed. A similar feature may be found in the definition of the generalized Lagrangian pseudomomentum (not to be confused with the generalized CE pseudomomentum of the present study) in AM78a. See Bühler (2009) for details. The

\footnotetext{
${ }^{5}$ Let an arbitrary quantity $A^{\prime}$ be associated with monochromatic waves so that $A^{\prime}$ is proportional to $\cos \theta$, where $\theta=k x-\sigma t$ is the wave phase. For example, in order to obtain the expression for the zonal component of the pseudomomentum vector in (17), we have substituted $A_{t}^{\prime} /(\sigma / k)=-k A_{\theta}^{\prime}=-A_{x}^{\prime}$ to each of $\xi_{t}^{\prime}, \eta_{t}^{\prime}$, and $\pi_{z t}^{\prime}$ in (15).
} 
generalized CE pseudomomentum vector in (17) reduces to $\langle\langle\bar{E} /(\sigma / k), \bar{E} /(\sigma / l)\rangle\rangle$ for waves that are nearly plane in the horizontal direction.
Using (8) and (16), we expand the zonal and meridional components of the generalized CE pseudomomentum vector in (17):

$$
\begin{aligned}
& \underbrace{\left(-u^{\prime} \xi_{x}^{\prime}-v^{\prime} \eta_{x}^{\prime}+\zeta^{\prime} \pi_{z x}^{\prime}\right) / 2}_{\text {CE pseudomomentum }}=\underbrace{\zeta_{z}^{\prime} u^{\prime}+q^{\prime} \eta^{\prime} / 2}_{\text {IB pseudomomentum }}+\left[\left(u^{\prime} \eta^{\prime}\right)_{y}-\left(v^{\prime} \eta^{\prime}\right)_{x}+\left(\zeta^{\prime} \pi_{x}^{\prime}\right)_{z}\right] / 2 \text { and } \\
& \underbrace{\left(-u^{\prime} \xi_{y}^{\prime}-v^{\prime} \eta_{y}^{\prime}+\zeta^{\prime} \pi_{z y}^{\prime}\right) / 2}_{\text {CE pseudomomentum }}=\underbrace{\zeta_{z}^{\prime} v^{\prime}-q^{\prime} \xi^{\prime} / 2}_{\text {IB pseudomomentum }}+\left[\left(v^{\prime} \xi^{\prime}\right)_{x}-\left(u^{\prime} \xi^{\prime}\right)_{y}+\left(\zeta^{\prime} \pi_{y}^{\prime}\right)_{z}\right] / 2,
\end{aligned}
$$

which have been little mentioned in previous studies (detailed derivation in the supplemental material). The time average of the first two terms on the right-hand sides of both (18a) and (18b) reads

$$
\left\langle\left\langle\overline{\zeta_{z}^{\prime} u^{\prime}}+\overline{q^{\prime} \eta^{\prime}} / 2, \overline{\zeta_{z}^{\prime} v^{\prime}}-\overline{q^{\prime} \xi^{\prime}} / 2\right\rangle\right\rangle,
$$

which is referred to as the impulse-bolus (IB) pseudomomentum vector in the present study, with the understanding that $\left\langle\left\langle\overline{q^{\prime} \eta^{\prime} / 2},-\overline{q^{\prime} \xi^{\prime} / 2}\right\rangle\right\rangle$ is a variant of the wave-impulse vector based on EPV, and $\left\langle\left\langle\overline{\zeta_{z}^{\prime} u^{\prime}}, \overline{\zeta_{z}^{\prime} v^{\prime}}\right\rangle\right\rangle$ is an Eulerian approximation for the bolus velocity (Rhines 1982; Gent et al. 1995) ( $\zeta_{z}^{\prime}$ may be interpreted as nondimensionalized thickness; see footnote 2$)$. The bolus velocity $\left\langle\left\langle\overline{\zeta_{z}^{\prime} u^{\prime}}, \overline{\zeta_{z}^{\prime} v^{\prime}}\right\rangle\right\rangle$ should not be confused with the quasi-Stokes velocity $\left\langle\left\langle u^{\mathrm{qs}}, v^{\mathrm{qs}}\right\rangle\right\rangle=\left\langle\left\langle\left(\overline{\zeta^{\prime} u^{\prime}}\right)_{z},\left(\overline{\zeta^{\prime} v^{\prime}}\right)_{z}\right\rangle\right\rangle$ in (13a) and (13b). The zonal component of the IB pseudomomentum vector $\overline{\zeta_{z}^{\prime} u^{\prime}+q^{\prime} \eta^{\prime} / 2}=\overline{\zeta_{z}^{\prime} u^{\prime}-\eta^{\prime 2 /(2 \beta)}}$ is identical to the pseudomomentum that has been suggested in Ripa (1982) and Andrews (1983a). A finiteamplitude wave version of this quantity has been developed by Haynes (1988) and Brunet and Haynes (1996) using the impulse-Casimir method assuming a zonally symmetric mean flow. The meridional component of the IB pseudomomentum vector has not been defined in previous studies because it is not a conserved quantity, as will be explained later in the paper (see section $2 \mathrm{~h}$ ).

The explicit relationship between the CE pseudomomentum vector and the IB pseudomomentum vector, as given by (18a) and (18b), is a cornerstone of the present study (Fig. 1). For example, (18a) and (18b) indicate that the volume integral of the IB pseudomomentum vector is identical to that of the CE pseudomomentum vector, assuming appropriate conditions for waves (i.e., either periodic or decaying) in the far field. It should also be noted that $\overline{u^{\prime} \xi^{\prime}} \simeq 0$ and $\overline{v^{\prime} \eta^{\prime}} \simeq 0$, owing to the phase relationship of neutral waves. This may provide a basis for the pseudomomentum of meridionally trapped EQWs to be understood using a cumulative sum in the meridional direction (to be explained later in the paper; see footnote 9).

\section{f. Relating the IB pseudomomentum vector to the wave-activity vector}

We have used the term wave activity in section 1 and the term pseudomomentum in section 2. The IB pseudomomentum vector may be related to the sum of the two types of wave activity associated with the gravity wave literature and the planetary wave literature (section 1), as follows. The difference between the quasiStokes velocity and the IB pseudomomentum reads

$\underbrace{\left(\overline{\zeta^{\prime} u^{\prime}}\right)_{z}}_{u^{\mathrm{qs}}}-\underbrace{\left(\overline{\zeta_{z}^{\prime} u^{\prime}}+\overline{\eta^{\prime} q^{\prime}} / 2\right)}_{\text {IB pseudomomentum }}=\underbrace{\overline{\zeta^{\prime} u_{z}^{\prime}}-\overline{\eta^{\prime} q^{\prime}} / 2}_{\text {DI wave activity }}$ and

$\underbrace{\left(\overline{\zeta^{\prime} v^{\prime}}\right)_{z}}_{v^{\mathrm{qs}}}-\underbrace{\left(\overline{\zeta_{z}^{\prime} v^{\prime}}-\overline{\xi^{\prime} q^{\prime}} / 2\right)}_{\text {IB pseudomomentum }}=\underbrace{\overline{\zeta^{\prime} v_{z}^{\prime}}+\overline{\xi^{\prime} q^{\prime}} / 2}_{\text {DI wave activity }}$,

which is referred to as the double-impulse (DI) wave activity in the present study (see also Fig. 1). This will be useful in section 4c.

The DI wave activity consists of two parts:

- The first part of the DI wave activity $\left\langle\left\langle\overline{\zeta^{\prime} u_{z}^{\prime}}, \overline{\zeta^{\prime} v_{z}^{\prime}}\right\rangle\right\rangle$ may be interpreted as a hydrostatic approximation for wave activity $\left\langle\left\langle\overline{\zeta^{\prime}\left(u_{z}^{\prime}-w_{x}^{\prime}\right)}, \overline{\zeta^{\prime}\left(v_{z}^{\prime}-w_{y}^{\prime}\right)}\right\rangle\right\rangle$ that has been used in the nonhydrostatic gravity wave literature (see supplemental material). See (6.16) of SS92 for the expression in the presence of the vertical shear of mean flows. A generalized expression for this part is $\left\langle\left\langle\overline{\int_{z}^{z+\zeta^{\prime}} u_{z} d z}, \overline{\left.\int_{z}^{z+\zeta^{\prime}} v_{z} d z\right\rangle}\right\rangle\right.$ for hydrostatic gravity waves and $\left\langle\left\langle\overline{\int_{z}^{z+\zeta^{\prime}}\left(u_{z}-w_{x}\right) d z}, \overline{\left.\left.\int_{z}^{z+\zeta^{\prime}}\left(v_{z}-w_{y}\right) d z\right\rangle\right\rangle}\right.\right.$ for nonhydrostatic gravity waves.

- The second part $\left\langle\left\langle-\overline{\eta^{\prime} q^{\prime}} / 2, \overline{\xi^{\prime} q^{\prime}} / 2\right\rangle\right\rangle$, in particular the zonal component, has been written at the second order in terms of a Taylor expansion in the direction of the horizontal gradient of the background $\mathrm{EPV}:-\eta^{\prime} q^{\prime} / 2=-\eta^{\prime} q^{\prime}-\left(\eta^{2} / 2\right) \bar{q}_{y} \simeq-\int_{y}^{y+\eta^{\prime}} q d y$, where 


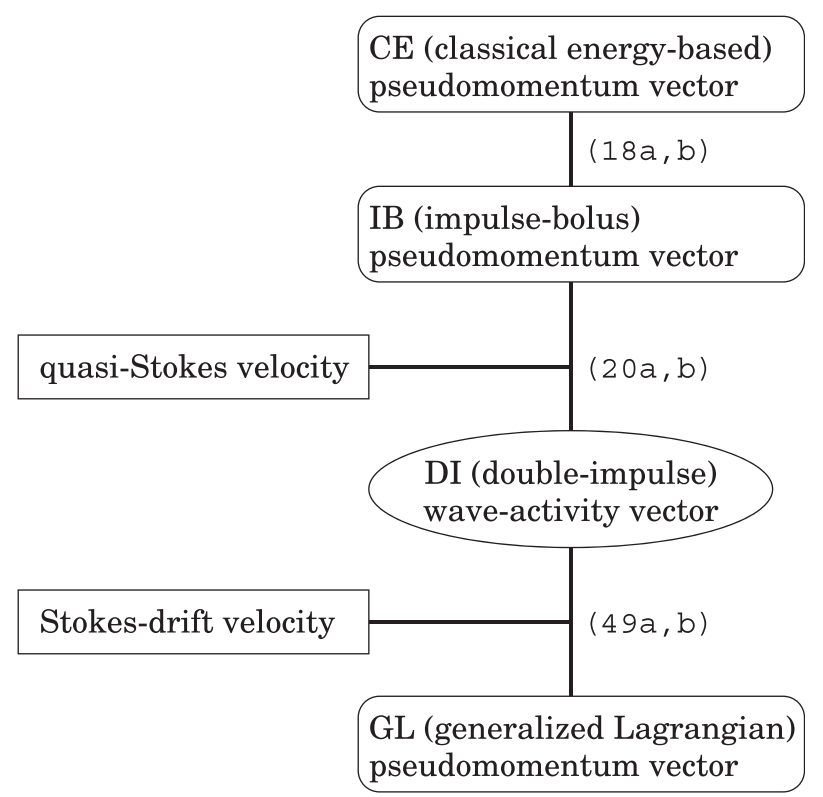

FIG. 1. Relationship of the pseudomomentum vectors, the waveactivity vector, and the wave-induced velocities in the present study.

$q^{\prime}+\eta^{\prime} \bar{q}_{y}=0$ has been used [see (11)]. This is why there is a factor of 2 in the denominator of this part (in contrast to the first part) in (20a) and (20b). This part of the DI wave activity has been used in the quasigeostrophic literature associated with either unstable quasigeostrophic waves (Bretherton 1966) or MRWs (Uryu 1974; AM76; P86). The expression of the meridional component $\overline{\xi^{\prime} q^{\prime}} / 2$ will prove useful in a future study in the presence of nonzero $\bar{q}_{x}$.

Equations (20a) and (20b) reconcile the difference in previous formulations between hydrostatic ageostrophic waves and nonhydrostatic gravity waves. The previous formulation of hydrostatic ageostrophic waves has adopted a shallow-water model or an isentropic coordinate system, which has led to the use of the bolus velocity as the pseudomomentum of gravity waves (i.e., the gravity wave part of ageostrophic pseudomomentum) (Ripa 1982; Andrews 1983a; Haynes 1988), as in (19). The previous formulation of nonhydrostatic gravity waves has adopted a vertical-slice model in a height (or pressure) coordinate system, which has led to the use of the impulse based on relative vorticity as the wave activity of gravity waves (SS92).

Principles for the definition of pseudomomentum/ wave activity in previous studies and the present study are summarized as follows. Most studies in atmospheric dynamics have adopted a zonal-mean framework, so pseudomomentum/wave activity may be regarded as a conserved scalar quantity. Then the form of pseudomomentum/wave activity has been determined from either quasigeostrophic dynamics, the impulseCasimir method, Hamiltonian dynamics, or Kelvin's circulation theorem (AM76; Haynes 1988; SS92; Ishioka and Yoden 1996; Bühler 2009; Nakamura and Solomon 2011; Solomon and Nakamura 2012; Methven 2013). On the other hand, the present study assumes that pseudomomentum/wave activity is a vector quantity, with an intent to develop a three-dimensional framework. Although we have not yet investigated its conservation property, we have already determined the form of the IB pseudomomentum vector from the explicit relationship in (18a) and (18b) with the CE pseudomomentum vector. This approach is similar in part to the definition of the generalized Lagrangian (GL) pseudomomentum vector in AM78a.

\section{g. Prognostic equations for the $C E$ pseudomomentum}

First, we note that taking the zonal derivative of (16a), (16b), and (9) yields

$$
\begin{aligned}
& \xi_{x t}^{\prime}-f \eta_{x}^{\prime}=-\pi_{x x}^{\prime}, \\
& \eta_{x t}^{\prime}+f \xi_{x}^{\prime}=-\pi_{y x}^{\prime}, \quad \text { and } \\
& \pi_{z x t}^{\prime}=p_{z x}^{\prime}=-N^{2} \zeta_{x}^{\prime},
\end{aligned}
$$

where $\left(N^{2}\right)_{x}=0$ is understood. Likewise, taking the meridional derivative of (16a), (16b), and (9) yields,

$$
\begin{gathered}
\xi_{y t}^{\prime}-f \eta_{y}^{\prime}-\beta \eta^{\prime}=-\pi_{x y}^{\prime}, \\
\eta_{y t}^{\prime}+f \xi_{y}^{\prime}+\beta \xi^{\prime}=-\pi_{y y}^{\prime}, \quad \text { and } \\
\pi_{z y t}^{\prime}=p_{z y}^{\prime}=-N^{2} \zeta_{y}^{\prime},
\end{gathered}
$$

where $f=f_{0}+\beta y$ and $\left(N^{2}\right)_{y}=0$ are understood.

We now derive a prognostic equation for the zonal component of the CE pseudomomentum vector in (17). Multiplying (6a), (6b), (21c), (21a), (21b), and $\zeta_{t}^{\prime}=w^{\prime}$ by $-\xi_{x}^{\prime} / 2,-\eta_{x}^{\prime} / 2, \zeta^{\prime} / 2,-u^{\prime} / 2,-v^{\prime} / 2$, and $\pi_{z x}^{\prime} / 2$, respectively, and then taking the sum of the six equations yields a prognostic equation for the zonal component of the CE pseudomomentum:

$$
[\underbrace{\left(-u^{\prime} \xi_{x}^{\prime}-v^{\prime} \eta_{x}^{\prime}+\zeta^{\prime} \pi_{z x}^{\prime}\right) / 2}_{\text {CE pseudomomentum }}]_{t}=-\nabla \cdot \underbrace{\left\langle\left\langle-\left(\xi_{x}^{\prime} p^{\prime}+u^{\prime} \pi_{x}^{\prime}\right) / 2,-\left(\eta_{x}^{\prime} p^{\prime}+v^{\prime} \pi_{x}^{\prime}\right) / 2,-\left(\zeta_{x}^{\prime} p^{\prime}+w^{\prime} \pi_{x}^{\prime}\right) / 2\right\rangle\right\rangle}_{\text {XCE flux }},
$$


which indicates that $\left(-u^{\prime} \xi_{x}^{\prime}-v^{\prime} \eta_{x}^{\prime}+\zeta^{\prime} \pi_{z x}^{\prime}\right) / 2$ is a conserved quantity (detailed derivation in the supplemental material). The "three dimensional" flux that appears inside the divergence operator in (23a) is herein referred to as the XCE flux or the CE flux. Next, we derive a prognostic equation for the meridional component of the CE pseudomomentum vector in (17). Multiplying (6a), (6b), (22c), (22a), (22b), and $\zeta_{t}^{\prime}=w^{\prime}$ by $-\xi_{y}^{\prime} / 2$, $-\eta_{y}^{\prime} / 2, \zeta^{\prime} / 2,-u^{\prime} / 2,-v^{\prime} / 2$, and $\pi_{z y}^{\prime} / 2$, respectively, and then taking the sum of the six equations yields a prognostic equation for the meridional component of the $\mathrm{CE}$ pseudomomentum:

$$
[\underbrace{\left(-u^{\prime} \xi_{y}^{\prime}-v^{\prime} \eta_{y}^{\prime}+\zeta^{\prime} \pi_{z y}^{\prime}\right) / 2}_{\text {CE pseudomomentum }}]_{t}=-\nabla \cdot \underbrace{\left\langle\left\langle-\left(\xi_{y}^{\prime} p^{\prime}+u^{\prime} \pi_{y}^{\prime}\right) / 2,-\left(\eta_{y}^{\prime} p^{\prime}+v^{\prime} \pi_{y}^{\prime}\right) / 2,-\left(\zeta_{y}^{\prime} p^{\prime}+w^{\prime} \pi_{y}^{\prime}\right) / 2\right\rangle\right\rangle}_{\text {YCE flux }}+\beta\left(v^{\prime} \xi^{\prime}-u^{\prime} \eta^{\prime}\right) / 2
$$

which indicates that $\left(-u^{\prime} \xi_{y}^{\prime}-v^{\prime} \eta_{y}^{\prime}+\zeta^{\prime} \pi_{z y}^{\prime}\right) / 2$ is not a conserved quantity owing to the planetary $\beta$ effect. ${ }^{6}$ The "three dimensional" flux that appears inside the divergence operator in (23b) is herein referred to as the YCE flux or the CE flux. So far, we have made no approximation specific to MIGWs, MRWs, and EQWs.

In the rest of this subsection, we investigate the direction of the CE flux in both (23a) and (23b) by specializing to waves that are nearly plane in the horizontal direction (such as MIGWs or MRWs; see Table 2). Let $A^{\prime}$ be an arbitrary quantity associated with (slowly varying) monochromatic waves that reads

$$
A^{\prime} \propto \cos \theta,
$$

where $\theta=k x+l y-\sigma t$ is wave phase, $\langle\langle k, l\rangle\rangle$ is the horizontal wavenumber vector, and $\sigma$ is wave frequency. Equation (24) yields

$$
\begin{aligned}
& A_{x}^{\prime} \simeq k A_{\theta}^{\prime}, \\
& A_{y}^{\prime} \simeq l A_{\theta}^{\prime}, \\
& A_{t}^{\prime} \simeq-\sigma A_{\theta}^{\prime}, \text { and } \\
& A_{\theta \theta}^{\prime}=-A^{\prime},
\end{aligned}
$$

where the approximated equality is associated with the slow variations of wave amplitude, $k, l$, and $\sigma$ (i.e., the WKB approximation). It should be noted that (24) and (25) provide no restriction for the vertical profile of waves, which enables, if necessary, the buoyancy frequency to vary in the vertical direction. Namely, it does

\footnotetext{
${ }^{6}$ This is not surprising. Previous studies based on the quasigeostrophic dynamics (Andrews 1984; P86; Takaya and Nakamura 1997; TN01) show that, if the pseudomomentum vector is projected onto the tangential and normal directions of the contours of EPV (i.e., the "pseudoeastward" and "pseudonorthward" directions) in the horizontal plane, only the tangential component is conserved, and the normal component is not.
}

not matter whether waves are nearly plane or nonplane in the vertical direction.

Substitution of both (25a)-(25d) and $\left\langle\left\langle\xi_{\theta}^{\prime}, \eta_{\theta}^{\prime}, \zeta_{\theta}^{\prime}\right\rangle\right\rangle \simeq$ $-\left\langle\left\langle u^{\prime}, v^{\prime}, w^{\prime}\right\rangle\right\rangle / \sigma$ to each of (23a) and (23b) yields,

$(E k / \sigma)_{t}+\nabla \cdot\left\langle\left\langle u^{\prime} p^{\prime} k / \sigma, v^{\prime} p^{\prime} k / \sigma, w^{\prime} p^{\prime} k / \sigma\right\rangle\right\rangle \simeq 0$ and

$(E l / \sigma)_{t}+\nabla \cdot\left\langle\left\langle u^{\prime} p^{\prime} l / \sigma, v^{\prime} p^{\prime} l / \sigma, w^{\prime} p^{\prime} l / \sigma\right\rangle\right\rangle \simeq \beta\left(v^{\prime} \xi^{\prime}-u^{\prime} \eta^{\prime}\right) / 2$,

which indicate that $E k / \sigma$ is a conserved quantity and $E l / \sigma$ is not a conserved quantity because of the planetary $\beta$ effect. Equations (26a) and (26b) allow for slow variations (in both the three-dimensional space and the time space) of the phase speeds $\sigma / k$ and $\sigma / l$, respectively. It should be noted that both (26a) and (26b) have been derived without using either the quasigeostrophic approximation (Andrews 1983b; P86) or the wave action and crest equations (Bretherton and Garrett 1968; Uryu 1974; Whitham 1974). This is attributed to the use of both the generalized expression of the CE pseudomomentum [(17)] and the instantaneous expression of prognostic equaions [(23a) and (23b)] (see footnote 4).

In terms of physical interpretation, however, (26a) and (26b) for the CE pseudomomentum have at least two problems. First, the CE flux in both (26a) and (26b) is proportional to the pressure flux $\left\langle\left\langle u^{\prime} p^{\prime}, v^{\prime} p^{\prime}, w^{\prime} p^{\prime}\right\rangle\right\rangle$ in (14) and thus is not, after application of a low-pass time filter, parallel to the group velocity of MRWs. Second, the quantities $\xi^{\prime}, \eta^{\prime}$, and $\pi^{\prime}$ in the CE pseudomomentum equations (23a) and (23b) are not readily available from model output. Likewise, $\sigma / k$ and $\sigma / l$ in (26a) and (26b) are not readily available from model output.

\section{h. Prognostic equations for the IB pseudomomentum}

We now derive a prognostic equation for the zonal component of the IB pseudomomentum in (19): 


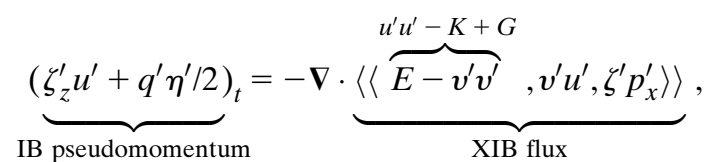

where (9)-(11) and $E=K+G$ have been used ( $K$ and $G$ are defined in section 2d; see a detailed derivation in the supplemental material). The "three dimensional" flux that appears inside the divergence operator in (27a) is herein referred to as the XIB flux or the IB flux. Equation (27a) indicates that $\left(\zeta_{z}^{\prime} u^{\prime}+q^{\prime} \eta^{\prime} / 2\right)$ is a conserved quantity and represents a generalized expression for the Eliassen-Palm relation [see (2)]. The zonal component of the IB pseudomomentum becomes, after application of a low-pass time filter and the use of (11), $\overline{\zeta_{z}^{\prime} u^{\prime}+q^{\prime} \eta^{\prime} / 2}=\left(\overline{\zeta^{\prime} u^{\prime}}\right)_{z}-\overline{\zeta^{\prime} u_{z}^{\prime}}-\overline{q^{\prime 2}} /(2 \beta)$, where the first two terms vanish ${ }^{7}$ under the combination of the quasigeostrophic approximation and the WKB approximation, and the last term corresponds to minus the wave activity $\overline{\overline{Q^{\dagger 2}}} /\left(2 \overline{\bar{Q}}_{y}\right)$ in (2). Likewise the meridional and vertical components of the IB flux in (27a) may be rewritten using (9) as $\left\langle\left\langle v^{\prime} u^{\prime}, \zeta^{\prime} p_{x}^{\prime}\right\rangle\right\rangle=$ $\left\langle\left\langle v^{\prime} u^{\prime},-\rho^{\prime} p_{x}^{\prime} / \bar{\rho}_{z}\right\rangle\right\rangle$, which corresponds, under the quasigeostrophic approximation, to minus the EliassenPalm flux $\left\langle\left\langle F^{y}, F^{z}\right\rangle\right\rangle$, as defined in (1b) and (1c). For all of MIGWs, MRWs, and EQWs, the zonal component of the IB pseudomomentum vector is conserved, which is as expected (Ripa 1982; Andrews 1983a; Haynes 1988). Indeed, both prototype and advanced forms of (27a) have been derived in previous studies, as listed in Table 3. In particular, the equations of Haynes (1988) and Brunet and Haynes (1996) have been derived using the impulse-Casimir method (assuming a zonally symmetric mean flow) and thus allow for the finite-amplitude undulation of the contours of EPV.

Next, we derive a prognostic equation for the meridional component of the IB pseudomomentum in (19), which has been little mentioned in previous studies:

\footnotetext{
${ }^{7}$ With application of a low-pass time filter and under the quasigeostrophic approximation, (27a) may be rewritten as $\left[\left(\overline{\zeta^{\prime} u^{\prime}}\right)_{z}+\left(\overline{\zeta^{\prime} p_{y z}^{\prime}}\right) /\right.$ $\left.f_{0}-\overline{q^{\prime 2}} /(2 \beta)\right]_{t}+\left(\overline{E-v^{\prime} v^{\prime}}\right)_{x}+\left(\overline{v^{\prime} u^{\prime}}\right)_{y}+\left(f_{0} \overline{\zeta^{\prime} v^{\prime}}\right)_{z} \simeq 0$. The second quantity in the tendency term vanishes as $\overline{\zeta^{\prime} p_{y z}^{\prime}} / f_{0}=-\overline{p_{z}^{\prime} p_{y z}^{\prime}} /\left(f_{0} N^{2}\right) \simeq$ $-\overline{p_{z}^{\prime} p_{\theta z}^{\prime}} l /\left(f_{0} N^{2}\right)=0$, where (9) and (25b) have been used. Then we compare the sizes of $\left(\overline{\zeta^{\prime} u^{\prime}}\right)_{z t}$ and $f_{0}\left(\overline{\zeta^{\prime} v^{\prime}}\right)_{z}$, the difference between which stems from the time scale of the slow variations of waves (not to be confused with the phase cycle of waves) and the inertial period. The former quantity scales out under the WKB approximation.
}

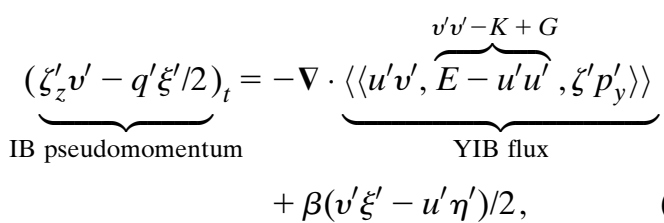

where (9)-(11) and $E=K+G$ have been used. The "three-dimensional" flux that appears inside the divergence operator in (27b) is herein referred to as the YIB flux or the IB flux. Equation (27b) indicates that $\left(\zeta_{z}^{\prime} v^{\prime}-q^{\prime} \xi^{\prime} / 2\right)$ is not a conserved quantity owing to the planetary $\beta$ effect. Meteorologists and oceanographers care whether a given expression for the pseudomomentum flux is suitable for model diagnosis (i.e., the expression being based on quantities that are readily available from model output). This criterion has been satisfied by the IB flux in each of (27a) and (27b). The meridional component of the IB pseudomomentum vector is not a conserved quantity but will be useful for a future study to diagnose the meridional propagation of equatorial inertia-gravity waves in the context of wavemean flow interaction.

Equations (27a) and (27b) may be rewritten using $q^{\prime}=-\beta \eta^{\prime}$ [i.e., (11)] as

$$
\left(\zeta_{z}^{\prime} u^{\prime}\right)_{t}+q^{\prime} v^{\prime}=-\nabla \cdot\left\langle\left\langle u^{\prime} u^{\prime}-K+G, v^{\prime} u^{\prime}, \zeta^{\prime} p_{x}^{\prime}\right\rangle\right\rangle \quad \text { and }
$$

$$
\left(\zeta_{z}^{\prime} v^{\prime}\right)_{t}-q^{\prime} u^{\prime}=-\nabla \cdot\left\langle\left\langle u^{\prime} v^{\prime}, v^{\prime} v^{\prime}-K+G, \zeta^{\prime} p_{y}^{\prime}\right\rangle\right\rangle,
$$

which involve the horizontal flux of EPV and thus represent a generalized expression for the Taylor-Bretherton identity (1a), including expressions used in (i) the midlatitude quasigeostrophic wave literature [Bretherton (1966), first equation on p. 329; P86, (2.18')] and (ii) the ageostrophic wave literature [Tung (1986); Hayashi and Young (1987); McPhaden and Ripa (1990); Takehiro and Hayashi (1992); see Table 3 of the present paper].

Both the IB pseudomomentum equations [(27a) and (27b)] and the generalized Taylor-Bretherton identity [(28a) and (28b)] are central to the present study and are applicable to the various types of linear hydrostatic neutral waves in a planetary fluid, such as MIGWs, MRWs, and EQWs. Although MIGWs are characterized by no perturbation of EPV ( $q^{\prime}=0$; Gill 1982; Müller 1995), the derivation of (27a) and (27b) remains the same, but letting $q^{\prime}=0$ and $\beta=0$.

The direction of the horizontal component of the IB flux may be explained as follows. For MIGWs, Miyahara (2006, hereafter M06) has shown at his (20) that the horizontal component of the IB flux in each of (27a) and (27b) is, after application of a low-pass time filter, parallel 
TABLE 3. List of the ageostrophic versions of the Taylor-Bretherton identity (1) and the Eliassen-Palm relation (2) in previous studies and the present study.

\begin{tabular}{lllc}
\hline \hline & Equation number & Zonal flux & Vertical flux \\
\hline Ageostrophic Taylor-Bretherton identity & & & \\
Tung (1986) & $(4.5)$ and (2.10) & Absent & Present \\
Hayashi and Young (1987) & $(2.28)$ & Absent & Absent \\
McPhaden and Ripa (1990) & $(22)$ & Absent & Absent \\
Takehiro and Hayashi (1992) & $(39)$ & Absent & Absent \\
This study & $(28 \mathrm{a})$ & Present & Present \\
Ageostrophic Eliassen-Palm relation & & & \\
Ripa (1982) & $(2.6 \mathrm{~d})$ & Present & Absent \\
Andrews (1983a) & $(4.1)$ & Absent & Present \\
Haynes (1988) & $(3.12 \mathrm{a})$ and (3.12b) & Present & Present \\
Brunet and Haynes (1996) & $(3.4 \mathrm{a})-(3.4 \mathrm{~d})$ & Present & Absent \\
This study & $(27 \mathrm{a})$ & Present & Present \\
\hline
\end{tabular}

to the group velocity of the waves. For MRWs, P86 has shown that the horizontal component of the XIB flux in (27a) is, after application of a low-pass time filter, parallel to the group velocity of the waves. ${ }^{8}$ For EQWs propagating in the zonal-vertical plane, substitution of both (25a) and (25c) into (6e) yields $k u_{\theta}^{\prime}+v_{y}^{\prime}-\sigma z_{z \theta}^{\prime} \simeq 0$, which indicates either $v^{\prime} \propto \cos \theta$ or $v^{\prime}=0$ if $u^{\prime} \propto \sin \theta$. Thus, $\overline{u^{\prime} v^{\prime}} \simeq 0$ : namely, the meridional component of the XIB flux in (27a) averages to zero. Further explanation for the characteristics of the IB flux associated with EQWs is given at the end of section 3a (see footnote 9).

On the other hand, the vertical component of the IB flux may be explained as follows for all of MIGWs, MRWs, and EQWs. Substitution of $\zeta^{\prime}=-\zeta_{\theta \theta}^{\prime} \simeq w_{\theta}^{\prime} / \sigma$ and (25a) into the vertical component of the XIB flux in (27a) yields $\overline{\zeta^{\prime} p_{x}^{\prime}} \simeq \overline{w_{\theta}^{\prime} p_{\theta}^{\prime}} k / \sigma=\overline{w^{\prime} p^{\prime}} k / \sigma$, where $\overline{\sin ^{2} \theta}=$ $\overline{\cos ^{2} \theta}$ has been used. Likewise, substitution of $\zeta^{\prime}=-\zeta_{\theta \theta}^{\prime} \simeq w_{\theta}^{\prime} / \sigma$ and $(25 \mathrm{~b})$ into the vertical component of the YIB flux in (27b) yields $\overline{\zeta^{\prime} p_{y}^{\prime}} \simeq \overline{w_{\theta}^{\prime} p_{\theta}^{\prime}} l / \sigma=\overline{w^{\prime} p^{\prime}} l / \sigma$. Thus each of $\overline{\zeta^{\prime} p_{x}^{\prime}}$ and $\overline{\zeta^{\prime} p_{y}^{\prime}}$ approximates to the vertical component of the CE flux.

The results of sections $2 \mathrm{~g}$ and $2 \mathrm{~h}$ may be summarized as follows. For MIGWs, both the CE and IB fluxes are parallel to the group velocity of waves. For MRWs, only the XIB flux is parallel to the group velocity of waves. For EQWs propagating in the zonal direction, the meridional component of both the XCE and XIB fluxes vanishes.

\footnotetext{
${ }^{8}$ Let the quasigeostrophic streamfunction be written by $\psi^{\prime} \propto \cos (k x+l y+m z-\sigma t)$, where $m$ is the vertical wavenumber. The group velocity of MRWs is written as $\left\langle\left\langle k^{2}-l^{2}-\left(f_{0} / N\right)^{2} m^{2}\right.\right.$, $\left.\left.2 k l, 2\left(f_{0} / N\right)^{2} k m\right\rangle\right\rangle \beta /\left[k^{2}+l^{2}+\left(f_{0} / N\right)^{2} m^{2}\right]^{2}$, where $N$ is assumed to be vertically uniform (Gill 1982; Holton 1992). The wave energy reduces to $E=(1 / 2)\left[k^{2}+l^{2}+\left(f_{0} / N\right)^{2} m^{2}\right] \psi^{\prime 2}$ under the assumption of quasigeostrophic monochromatic waves [i.e., $u^{\prime}=-\psi_{x}^{\prime}, v^{\prime}=\psi_{y}^{\prime}$, and $\left.\zeta^{\prime}=-\psi_{z}^{\prime} f_{0} / N^{2}\right]$. Thus, $\left\langle\left\langle\overline{E-v^{\prime} v^{\prime}}, \overline{v^{\prime} u^{\prime}}, \overline{\zeta^{\prime} p_{x}^{\prime}}\right\rangle\right\rangle$ is parallel to the group velocity of MRWs $\left(p^{\prime}=\psi^{\prime} f_{0}\right.$ is understood).
}

\section{Origin of the difference in the direction of the CE and IB fluxes}

In this section, we investigate what makes the threedimensional fluxes in the CE pseudomomentum equations (23a) and (23b) and the IB pseudomomentum equations (27a) and (27b) look in different directions. We show that the CE and IB fluxes are linked by a gauge transformation, previously unmentioned, associated with the divergence-form wave-induced pressure $\Lambda$. Then, we present two approaches for estimating $\Lambda$ to understand how the characteristics of it vary depending on MIGWs, MRWs, and EQWs.

\section{a. Gauge transformation between the $C E$ and $I B$ pseudomomentum equations}

It is of interest to identify the origin of the difference in the direction of the CE flux (section $2 \mathrm{~g}$ ) and the IB flux (section 2h). First, we rewrite each component of the CE flux in each of the CE pseudomomentum equations (23a) and (23b):

$$
\begin{aligned}
& -\left(\xi_{x}^{\prime} p^{\prime}+u^{\prime} \pi_{x}^{\prime}\right) / 2=\left(\xi^{\prime} p_{x}^{\prime}-u^{\prime} \pi_{x}^{\prime}\right) / 2-\left(\xi^{\prime} p^{\prime} / 2\right)_{x}, \\
& -\left(\eta_{x}^{\prime} p^{\prime}+v^{\prime} \pi_{x}^{\prime}\right) / 2=\left(\eta^{\prime} p_{x}^{\prime}-v^{\prime} \pi_{x}^{\prime}\right) / 2-\left(\eta^{\prime} p^{\prime} / 2\right)_{x}, \\
& -\left(\xi_{x}^{\prime} p^{\prime}+w^{\prime} \pi_{x}^{\prime}\right) / 2=\left(\zeta^{\prime} p_{x}^{\prime}-w^{\prime} \pi_{x}^{\prime}\right) / 2-\left(\xi^{\prime} p^{\prime} / 2\right)_{x}, \\
& -\left(\xi_{y}^{\prime} p^{\prime}+u^{\prime} \pi_{y}^{\prime}\right) / 2=\left(\xi^{\prime} p_{y}^{\prime}-u^{\prime} \pi_{y}^{\prime}\right) / 2-\left(\xi^{\prime} p^{\prime} / 2\right)_{y}, \\
& -\left(\eta_{y}^{\prime} p^{\prime}+v^{\prime} \pi_{y}^{\prime}\right) / 2=\left(\eta^{\prime} p_{y}^{\prime}-v^{\prime} \pi_{y}^{\prime}\right) / 2-\left(\eta^{\prime} p^{\prime} / 2\right)_{y}, \quad \text { and } \\
& -\left(\zeta_{y}^{\prime} p^{\prime}+w^{\prime} \pi_{y}^{\prime}\right) / 2=\left(\zeta^{\prime} p_{y}^{\prime}-w^{\prime} \pi_{y}^{\prime}\right) / 2-\left(\zeta^{\prime} p^{\prime} / 2\right)_{y} .
\end{aligned}
$$

We substitute (29a)-(29c) to the zonal component of the CE pseudomomentum equation (23a) and then single out the quantity $\Lambda \equiv\left[\left(\xi^{\prime} p^{\prime}\right)_{x}+\left(\eta^{\prime} p^{\prime}\right)_{y}+\left(\zeta^{\prime} p^{\prime}\right)_{z}\right] / 2$ to yield 
$\left[(\underbrace{\left.-u^{\prime} \xi_{x}^{\prime}-v^{\prime} \eta_{x}^{\prime}+\zeta^{\prime} \pi_{z x}^{\prime}\right) / 2}]_{t}=-\nabla \cdot\left\langle\left\langle\left(\xi^{\prime} p_{x}^{\prime}-u^{\prime} \pi_{x}^{\prime}\right) / 2-\Lambda,\left(\eta^{\prime} p_{x}^{\prime}-v^{\prime} \pi_{x}^{\prime}\right) / 2,\left(\zeta^{\prime} p_{x}^{\prime}-w^{\prime} \pi_{x}^{\prime}\right) / 2\right\rangle\right\rangle\right.$,

CE pseudomomentum

where the direction of the three-dimensional flux (which appears inside the divergence operator) has been changed from that in (23a), although the divergence of the flux remains the same. Likewise, we substitute (29d)(29f) to the meridional component of the CE pseudomomentum equation (23b) and then single out $\Lambda$ to yield

$$
[\underbrace{\left(-u^{\prime} \xi_{y}^{\prime}-v^{\prime} \eta_{y}^{\prime}+\zeta^{\prime} \pi_{z y}^{\prime}\right) / 2}_{\text {CE pseudomomentum }}]_{t}=-\nabla \cdot\left\langle\left\langle\left(\xi^{\prime} p_{y}^{\prime}-u^{\prime} \pi_{y}^{\prime}\right) / 2,\left(\eta^{\prime} p_{y}^{\prime}-v^{\prime} \pi_{y}^{\prime}\right) / 2-\Lambda,\left(\zeta^{\prime} p_{y}^{\prime}-w^{\prime} \pi_{y}^{\prime}\right) / 2\right\rangle\right\rangle+\beta\left(v^{\prime} \xi^{\prime}-u^{\prime} \eta^{\prime}\right),
$$

where the direction of the three-dimensional flux (which appears inside the divergence operator) has been changed from that in (23b), although, as before, the divergence of the flux remains the same.

Below we show that the modified forms of the CE pseudomomentum equations (30a) and (30b) are closely related with the IB pseudomomentum (27a) and (27b). First, we multiply (6a), (6b), and (9) by $\xi^{\prime}, \eta^{\prime}$, and $N^{2} \zeta^{\prime}$, respectively, and then take the sum of the three equations to yield

$$
\begin{aligned}
& \left(\xi^{\prime} u_{t}^{\prime}+\eta^{\prime} v_{t}^{\prime}\right)-f\left(\xi^{\prime} v^{\prime}-\eta^{\prime} u^{\prime}\right) \\
& \quad+\xi^{\prime} p_{x}^{\prime}+\eta^{\prime} p_{y}^{\prime}+\zeta^{\prime} p_{z}^{\prime}+N^{2} \zeta^{\prime 2}=0 .
\end{aligned}
$$

Equation (31) may be written as

$$
\Lambda=-E+\left(u^{\prime} u^{\prime}-\xi^{\prime} u_{t}^{\prime}+v^{\prime} v^{\prime}-\eta^{\prime} v_{t}^{\prime}\right) / 2+f\left(\xi^{\prime} v^{\prime}-\eta^{\prime} u^{\prime}\right) / 2,
$$

where $E \equiv\left(u^{\prime 2}+v^{\prime 2}+N^{2} \zeta^{\prime 2}\right) / 2$ should be understood. Using (32), we now investigate each component of the three-dimensional flux in the modified forms of the CE pseudomomentum equations (30a) and (30b):

$$
\begin{aligned}
\left(\xi^{\prime} p_{x}^{\prime}-u^{\prime} \pi_{x}^{\prime}\right) / 2-\Lambda & =E-v^{\prime} v^{\prime}+\left(v^{\prime} \eta^{\prime}\right)_{t} / 2, \\
\left(\eta^{\prime} p_{x}^{\prime}-v^{\prime} \pi_{x}^{\prime}\right) / 2 & =v^{\prime} u^{\prime}-\left(u^{\prime} \eta^{\prime}\right)_{t} / 2, \\
\left(\zeta^{\prime} p_{x}^{\prime}-w^{\prime} \pi_{x}^{\prime}\right) / 2 & =\zeta^{\prime} p_{x}^{\prime}-\left(\zeta^{\prime} \pi_{x}^{\prime}\right)_{t} / 2, \\
\left(\xi^{\prime} p_{y}^{\prime}-u^{\prime} \pi_{y}^{\prime}\right) / 2 & =u^{\prime} v^{\prime}-\left(v^{\prime} \xi^{\prime}\right)_{t} / 2, \\
\left(\eta^{\prime} p_{y}^{\prime}-v^{\prime} \pi_{y}^{\prime}\right) / 2-\Lambda & =E-u^{\prime} u^{\prime}+\left(u^{\prime} \xi^{\prime}\right)_{t} / 2, \quad \text { and } \\
\left(\zeta^{\prime} p_{y}^{\prime}-w^{\prime} \pi_{y}^{\prime}\right) / 2 & =\zeta^{\prime} p_{y}^{\prime}-\left(\zeta^{\prime} \pi_{y}^{\prime}\right)_{t} / 2,
\end{aligned}
$$

where (33a) and (33e) have been derived using (6a) and (6b), (16a) and (16b), and (32) (detailed derivation in the supplemental material). We now substitute (33a)(33f) into the modified forms of the CE pseudomomentum equations (30a) and (30b) to yield

$$
\begin{aligned}
& {[\underbrace{\left(-u^{\prime} \xi_{x}^{\prime}-v^{\prime} \eta_{x}^{\prime}+\zeta^{\prime} \pi_{z x}^{\prime}\right) / 2}_{\text {CE pseudomomentum }}]_{t}=-\nabla \cdot\left\langle\left\langle E-v^{\prime} v^{\prime}+\left(v^{\prime} \eta^{\prime}\right)_{t} / 2, v^{\prime} u^{\prime}-\left(u^{\prime} \eta^{\prime}\right)_{t} / 2, \zeta^{\prime} p_{x}^{\prime}-\left(\zeta^{\prime} \pi_{x}^{\prime}\right)_{t} / 2\right\rangle\right\rangle \quad \text { and }} \\
& {[\underbrace{\left(-u^{\prime} \xi_{y}^{\prime}-v^{\prime} \eta_{y}^{\prime}+\zeta^{\prime} \pi_{z y}^{\prime}\right) / 2}_{\text {CE pseudomomentum }}]_{t}=-\nabla \cdot\left\langle\left\langle u^{\prime} v^{\prime}-\left(v^{\prime} \xi^{\prime}\right)_{t} / 2, E-u^{\prime} u^{\prime}+\left(u^{\prime} \xi^{\prime}\right)_{t} / 2, \zeta^{\prime} p_{y}^{\prime}-\left(\zeta^{\prime} \pi_{y}^{\prime}\right)_{t} / 2\right\rangle\right\rangle+\beta\left(v^{\prime} \xi^{\prime}-u^{\prime} \eta^{\prime}\right) / 2 .}
\end{aligned}
$$

It is clear that moving all terms with time derivatives on the right-hand sides of (34a) and (34b) to the lefthand side will lead to reproduction of the IB pseudomomentum equations (27a) and (27b), understanding the explicit relationship between the CE pseudomomentum and the IB pseudomomentum in (18a) and (18b). We conclude that the quantity $\Lambda \equiv\left[\left(\xi^{\prime} p^{\prime}\right)_{x}+\right.$ $\left.\left(\eta^{\prime} p^{\prime}\right)_{y}+\left(\zeta^{\prime} p^{\prime}\right)_{z}\right] / 2$ is at the heart of the difference in the direction of the CE and IB fluxes, which has been little mentioned in previous studies. It can be said that the IB and CE fluxes are linked by a gauge transformation associated with $\Lambda$. The quantity $\Lambda$ is also 
useful for understanding the characteristics of the XIB flux associated with EQWs propagating in the zonalvertical plane. ${ }^{9}$

In the rest of this section, we investigate the characteristics of $\Lambda$ for MIGWs, MRWs, and EQWs. In particular, we suggest two approaches for estimating $\Lambda$. The first approach is based on the combination of the virial theorem and the potential vorticity equation (section $3 b$ ). The second approach is based on the assumption of nearly plane waves in the horizontal direction (section 3c).

\section{b. First approach to estimate $\Lambda$ : The combination of the virial theorem and the potential vorticity equation}

For readers who are unfamiliar with the virial theorem, we begin with noting a well-known equipartition statement between the wave kinetic energy $\bar{K}$ and the wave potential energy $\bar{G}$ associated with linear waves in a nonrotating frame (Bühler 2009). The equipartition statement may be shown by manipulating (31) to yield

$$
\begin{aligned}
& {\left[\left(\xi^{\prime} u^{\prime}+\eta^{\prime} v^{\prime}\right) / 2\right]_{t}-f\left(\xi^{\prime} v^{\prime}-\eta^{\prime} u^{\prime}\right) / 2} \\
& +\underbrace{\left[\left(\xi^{\prime} p^{\prime}\right)_{x}+\left(\eta^{\prime} p^{\prime}\right)_{y}+\left(\xi^{\prime} p^{\prime}\right)_{z}\right] / 2}_{\equiv \Lambda}+G=K .
\end{aligned}
$$

If steady waves in a periodic nonrotating domain are considered, the first three terms on the left-hand side of (35) vanish after application of a low-pass time filter. The result is that $\bar{G}$ becomes equal to $\bar{K}$. Equation (35) may be referred to as an Eulerian expression for the virial theorem.

For waves in a rotating frame, Andrews and McIntyre (1978b, hereafter AM78b) have used the virial theorem to explain the concept of generalized wave action. AM78b have eventually focused on three-dimensionally homogeneous waves (i.e., waves other than planetary waves) to ignore $\bar{\Lambda}$. This may be confirmed by noting

\footnotetext{
${ }^{9}$ Substitution of (33a) to the zonal component of the XIB flux in (27a) yields $E-v^{\prime} v^{\prime}=-\Lambda+\left(\xi^{\prime} p_{x}^{\prime}-u^{\prime} \pi_{x}^{\prime}\right) / 2-\left(\eta^{\prime} v^{\prime}\right)_{t}=-\left(\xi_{x}^{\prime} p^{\prime}+\right.$ $\left.u^{\prime} \pi_{x}^{\prime}\right) / 2-\left[\left(\eta^{\prime} p^{\prime}\right)_{y}+\left(\zeta^{\prime} p^{\prime}\right)_{z}+\left(\eta^{\prime} v^{\prime}\right)_{t}\right] / 2$, where the $-\left(\xi_{x}^{\prime} p^{\prime}+u^{\prime} \pi_{x}^{\prime}\right) / 2$ part is identical to the zonal component of the XCE flux in (23a) and thus approximates to $u^{\prime} p^{\prime} k / \sigma$, as in (26a). The time average of the remaining part $\overline{\left[\left(\eta^{\prime} p^{\prime}\right)_{y}+\left(\zeta^{\prime} p^{\prime}\right)_{z}+\left(\eta^{\prime} v^{\prime}\right)_{t}\right]} / 2 \simeq$ $\left[\overline{\left(\eta^{\prime} p^{\prime}\right)_{y}+\left(\zeta^{\prime} p^{\prime}\right)_{z}}\right] / 2$ vanishes after taking an areal integral in the meridional-vertical plane, assuming meridionally and vertically trapped waves. To summarize, for EQWs propagating in the zonal-vertical plane, an areal integral in the meridional and vertical section is understood when discussing the relationship between the XIB flux and the group velocity of the waves, via the pressure flux in the wave energy equation (14). This feature originates from (18a), where the difference between the CE pseudomomentum and the IB pseudomomentum is defined as a flux divergence form.
}

that $2 \bar{\Lambda}$ in the present study corresponds to $(1 / \bar{\rho}) \overline{\left(\xi_{j} p^{\prime}\right)_{, j}}$ in (B2) of AM78b. Likewise, $2(\bar{\Lambda}+\bar{G})$ in the present study corresponds to $(1 / \tilde{\rho}) \overline{\xi_{i} K_{i j}\left(p^{\xi}\right)_{j}}$ in (4.10) of AM78b. On the other hand, Eckart (1963) has used the virial theorem to consider the stability problem of a mean flow. He has eventually removed $\bar{\Lambda}$ by taking a volume integral.

We suggest that the virial theorem, as expressed by (35), is actually applicable to all waves at all latitudes as long as $\Lambda$ is retained. Substitution of the potential vorticity equation (11) and (25c) and (25d) into (35) and then application of a low-pass time filter yields

$$
\bar{\Lambda} \simeq \bar{K}-\bar{G}+(f / \beta) \overline{q^{\prime} u^{\prime}},
$$

which allows us to estimate $\bar{\Lambda}$ analytically and numerically. Because $\eta^{\prime}=-q^{\prime} / \beta$ has been used, (36) is applicable to both EQWs and MRWs, but not MIGWs (Table 2). It should be noted that (36) provides no restriction for the vertical profile of waves, which enables, if necessary, the buoyancy frequency to vary in the vertical direction. Namely, it does not matter whether waves are nearly plane or nonplane in the vertical direction. For EQWs (which have a trapped modal structure in the meridional direction), one may easily expect that $\left(\overline{\eta^{\prime} p^{\prime}}\right)_{y} \neq 0$ and thus anticipate that $\bar{\Lambda}$ is nonzero. On the other hand, for MRWs, $\bar{\Lambda}$ must be nonzero in order for the $\mathrm{CE}$ and IB fluxes to look in different directions, but this is not obvious from (36). To summarize, while (36) will be useful for the model diagnosis of $\bar{\Lambda}$ in a future study, it is not so useful for interpreting $\bar{\Lambda}$.

\section{c. Second approach to estimate $\Lambda$ : The assumption of nearly plane waves in the horizontal direction}

We develop another equation to diagnose $\Lambda$ :

$$
\begin{aligned}
\Lambda= & \left(\xi^{\prime} p_{x}^{\prime}+\eta^{\prime} p_{y}^{\prime}+\zeta^{\prime} p_{z}^{\prime}\right) / 2 \\
= & \left(\xi^{\prime} p_{x}^{\prime}+\eta^{\prime} p_{y}^{\prime}\right) / 2-\left(N^{2} / 2\right) \zeta^{\prime 2} \\
= & {\left[-\left(\pi_{y}^{\prime}+v^{\prime}\right) p_{x}^{\prime}+\left(\pi_{x}^{\prime}+u^{\prime}\right) p_{y}^{\prime}\right] /(2 f)-\left(N^{2} / 2\right) \zeta^{\prime 2} } \\
= & {\left[-\pi_{y}^{\prime} p_{x}^{\prime}+\pi_{x}^{\prime} p_{y}^{\prime}\right] /(2 f) } \\
& +\left[v^{\prime}\left(-p_{x}^{\prime}+f v^{\prime}\right)+u^{\prime}\left(p_{y}^{\prime}+f u^{\prime}\right)\right] /(2 f)-E
\end{aligned}
$$

where the first line has been derived using (8), the second line has been derived using (9), the third line has been derived using (16a) and (16b), and the last line has been derived using $E \equiv\left(u^{\prime 2}+v^{\prime 2}+N^{2} \zeta^{\prime 2}\right) / 2$. It should be noted that (37) provides no restriction for the vertical profile of waves, which enables, if necessary, the buoyancy frequency $N$ to vary in the vertical direction. Namely, it does not matter whether waves are nearly plane or nonplane in the vertical direction. 
An expression for $\bar{\Lambda}$ that is suitable for analytical interpretation may be obtained by substituting (6a) and (6b) into the right-hand side of (37) and then applying a low-pass time filter to yield

$$
\bar{\Lambda}=\underbrace{\left({\overline{\left(-\pi_{y}^{\prime} p_{x}^{\prime}+\pi_{x}^{\prime} p_{y}^{\prime}\right.}}_{)}\right) /(2 f)}_{\simeq 0 \text { for plane waves }}+\underbrace{\overbrace{\left(\overline{u_{t}^{\prime} v^{\prime}-u^{\prime} v_{t}^{\prime}}\right) /(2 f)}^{\simeq \text { for MIGWs }}}_{\simeq 0 \text { for MRWs }}-\bar{E} .
$$

The first term on the right-hand side of (38) vanishes for both MIGWs and MRWs (but it is nonzero for EQWs, as will be explained later in the next paragraph). This is because the assumption of nearly plane waves allows (25a) (25d) to be used to yield $\left(\overline{-\pi_{y}^{\prime} p_{x}^{\prime}+\pi_{x}^{\prime} p_{y}^{\prime}}\right) /(2 f) \simeq$ $\left(\overline{\pi_{\theta}^{\prime} \pi_{\theta \theta}^{\prime}-\pi_{\theta}^{\prime} \pi_{\theta \theta}^{\prime}}\right) k l \sigma /(2 f)=0$. The characteristics of the second term on the right-hand side of (38) vary depending on MIGWs and MRWs. For MIGWs, substitution of a standard analytical solution for threedimensional plane waves (wherein $N$ is assumed to be a vertically uniform constant; see appendix A) to the term leads to $\left(\overline{u_{t}^{\prime} v^{\prime}-u^{\prime} v_{t}^{\prime}}\right) /(2 f) \simeq \bar{E}$. On the other hand, for MRWs, $\left(\overline{u_{t}^{\prime} v^{\prime}-u^{\prime} v_{t}^{\prime}}\right) /(2 f) \simeq\left(\overline{-p_{y t}^{\prime} p_{x}^{\prime}+p_{y}^{\prime} p_{x t}^{\prime}}\right) /$ $\left(2 f^{2}\right) \simeq\left(\overline{p_{\theta \theta}^{\prime} p_{\theta}^{\prime}-p_{\theta}^{\prime} p_{\theta \theta}^{\prime}}\right) k l \sigma /\left(2 f^{2}\right)=0$, where both geostrophic velocity and (25a)-(25d) have been used. To summarize, (38) yields $\bar{\Lambda} \simeq 0$ for MIGWs and $\bar{\Lambda} \simeq-\bar{E}$ for MRWs (Table 2). In section 3a, we have explained that $\bar{\Lambda}$ is at the heart of the difference in the direction of the CE flux in (23a) and (23b) and the IB flux in (27a) and (27b), referencing (30) and (33a)-(33f). That $\bar{\Lambda} \simeq 0$ for MIGWs (at least for uniform $N$ ) is surely important and worth emphasizing. This is why the CE flux in (23a) and (23b) and the IB flux in (27a) and (27b) both are parallel to the direction of the group velocity for MIGWs. That $\bar{\Lambda}$ is nonzero for MRWs means that these two fluxes point in different directions in that case, and since the IB flux in (27a) is parallel to the direction of the group velocity for MRWs (see footnote 8), the CE flux in (23a) cannot be.

On the other hand, for EQWs, the characteristics of both the first and second terms on the right-hand side of (38) are unclear. Nevertheless, for EQWs (and MRWs), we have already explained that (36) may be used. See also footnote 9 . The above-mentioned approaches [i.e., the set of (36) and (37)] to estimate $\bar{\Lambda}$ are complementary to each other and have been developed for the understanding of the difference in the direction of the $\mathrm{CE}$ and IB fluxes. The two approaches are also useful, via the virial theorem (35), for the estimation of the quantity $\left(\overline{\xi^{\prime} v^{\prime}-\eta^{\prime} u^{\prime}}\right) / 2 \simeq \overline{\xi^{\prime} v^{\prime}} \simeq-\overline{\eta^{\prime} u^{\prime}}$ associated with the Stokes-drift velocity in (12a) and (12b) (appendix B and Table 2).
To summarize this section, we have investigated what makes the CE and IB fluxes look in different directions. Since the CE and IB pseudomomenta only differ by divergence of a vector [see (18a) and (18b)], their prognostic equations are related through the gauge transformation associated with the divergence-form wave-induced pressure $\Lambda$, which is the most important result of the present study (section 3a). Then we have investigated the characteristics of $\Lambda$ for MIGWs, MRWs, and EQWs, with two approaches for estimating $\Lambda$. The first approach is based on the combination of the virial theorem and the potential vorticity equation and is applicable to MRWs and EQWs (section 3b). The second approach is based on the assumption of nearly plane waves in the horizontal direction and is applicable to MIGWs and MRWs (section 3c).

One of the reasons why we have used the CE flux as a reference for affirming the direction of the IB flux is that the CE flux is proportional to not only the pressure flux in the wave energy equation, but also the threedimensional form stress in the three-dimensional LM momentum equations (see section 4a). Namely, the three-dimensional form stress is parallel to the direction of the group velocity of MIGWs, but not for MRWs, as explained in the next section.

\section{The effect of waves on the mean flow}

Using analytical solutions for MIGWs and MRWs, M06 and Kinoshita and Sato (2013, hereafter KS13) have derived three-dimensional versions of the TEM momentum equation (3) and then examined whether the wave-induced momentum flux on the right-hand side of their TEM momentum equations is parallel to the group velocity of waves. In both studies, the Coriolis term of their TEM momentum equations has been written in terms of the LM velocity: namely, the sum of the EM velocity and the Stokes-drift velocity [see (12a) and (12b)]. Noda (2010) has shown, in a general way, a basis for writing the Coriolis term using the LM velocity, which is revisited in section 4 a as a preliminary discussion for this section.

In section $4 \mathrm{~b}$, we derive a generalized version of the TEM momentum equation (3), in which the effect of waves on the mean flow is found to be represented by the set of the Coriolis-Stokes force, the threedimensional divergence of the IB flux, and the horizontal gradient of $\bar{\Lambda}$. This indicates another utility of $\bar{\Lambda}$ that has been little mentioned in previous studies. In section $4 \mathrm{c}$, we derive a generalized version of the MEM momentum equation (4), in which the prognostic quantity is found to be the sum of the EM velocity and the DI wave activity in (20a) and (20b). All equations in 

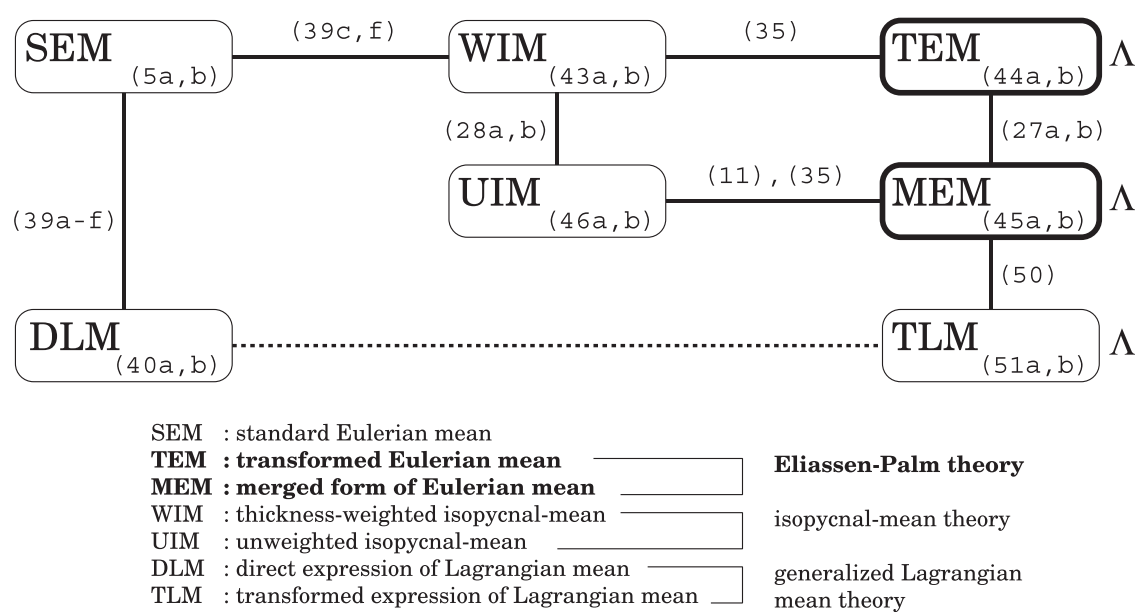

FIG. 2. Relationship of the seven sets of low-pass time-filtered momentum equations in the present study: SEM is the standard EM momentum equations (5a) and (5b), DLM is (an Eulerian approximation for) the direct expression of the three-dimensional LM momentum equations (40a) and (40b), WIM is (an Eulerian approximation for) the thicknessweighted isopycnal-mean momentum equations (43a) and (43b), TEM is the generalized transformed EM momentum equations (44a) and (44b), MEM is the merged form of the EM momentum equations (45a) and (45b), UIM is (an Eulerian approximation for) the unweighted isopycnal-mean momentum equations (46a) and (46b) in a vector-invariant form, and TLM is (an Eulerian approximation for) the transformed expression of the three-dimensional LM momentum equations (51a) and (51b). The set of TEM and MEM originates from the Eliassen-Palm theory, as noted by thick boxes. The set of WIM and UIM originates from the isopycnal-mean theory. The set of DLM and TLM originates from the generalized LM theory. The quantity $\Lambda$ is found in each of the TEM, MEM, and TLM momentum equations.

sections $4 \mathrm{~b}$ and $4 \mathrm{c}$ are applicable to MIGWs, MRWs, and EQWs.

\section{a. Low-pass-filtered momentum equations based on the three-dimensional form stress}

In the standard EM momentum equations (5a) and $(5 b)$, the effect of waves on the mean flow has been represented by the three-dimensional Reynolds stress, which we wish to transform using (6a), (6b), and (7):

$$
\begin{aligned}
& u^{\prime} u^{\prime}=\left(\xi^{\prime} u^{\prime}\right)_{t}-\xi^{\prime} u_{t}^{\prime}=\left(\xi^{\prime} u^{\prime}\right)_{t}+\xi^{\prime} p_{x}^{\prime}-f \xi^{\prime} v^{\prime} \\
& v^{\prime} u^{\prime}=\left(\eta^{\prime} u^{\prime}\right)_{t}-\eta^{\prime} u_{t}^{\prime}=\left(\eta^{\prime} u^{\prime}\right)_{t}+\eta^{\prime} p_{x}^{\prime}-f \eta^{\prime} v^{\prime} \\
& w^{\prime} u^{\prime}=\left(\zeta^{\prime} u^{\prime}\right)_{t}-\zeta^{\prime} u_{t}^{\prime}=\left(\zeta^{\prime} u^{\prime}\right)_{t}+\zeta^{\prime} p_{x}^{\prime}-f \zeta^{\prime} v^{\prime} \\
& u^{\prime} v^{\prime}=\left(\xi^{\prime} v^{\prime}\right)_{t}-\xi^{\prime} v_{t}^{\prime}=\left(\xi^{\prime} v^{\prime}\right)_{t}+\xi^{\prime} p_{y}^{\prime}+f \xi^{\prime} u^{\prime} \\
& v^{\prime} v^{\prime}=\left(\eta^{\prime} v^{\prime}\right)_{t}-\eta^{\prime} v_{t}^{\prime}=\left(\eta^{\prime} v^{\prime}\right)_{t}+\eta^{\prime} p_{y}^{\prime}+f \eta^{\prime} u^{\prime} \\
& w^{\prime} v^{\prime}=\left(\zeta^{\prime} v^{\prime}\right)_{t}-\zeta^{\prime} v_{t}^{\prime}=\left(\zeta^{\prime} v^{\prime}\right)_{t}+\zeta^{\prime} p_{y}^{\prime}+f \zeta^{\prime} u^{\prime}
\end{aligned}
$$

Substitution of (39a)-(39f) to the standard EM momentum equations (5a) and (5b) yields (Fig. 2)

$$
\begin{aligned}
& \left(\bar{u}+u^{\text {Stokes }}\right)_{t}+\nabla \cdot(\overline{\mathbf{U}} \bar{u})-f\left(\bar{v}+v^{\text {Stokes }}\right)-\beta\left(\overline{\eta^{\prime} v^{\prime}}\right) \\
& \quad=-\bar{p}_{x}-\nabla \cdot\left\langle\left\langle\overline{\xi^{\prime} p_{x}^{\prime}}, \overline{\eta^{\prime} p_{x}^{\prime}}, \overline{\zeta^{\prime} p_{x}^{\prime}}\right\rangle\right\rangle \text { and } \\
& \left(\bar{v}+v^{\text {Stokes }}\right)_{t}+\nabla \cdot(\overline{\mathbf{U}} \bar{v})+f\left(\bar{u}+u^{\text {Stokes }}\right)+\beta\left(\overline{\eta^{\prime} u^{\prime}}\right) \\
& \quad=-\bar{p}_{y}-\nabla \cdot\left\langle\left\langle\overline{\xi^{\prime} p_{y}^{\prime}}, \overline{\eta^{\prime} p_{y}^{\prime}}, \overline{\zeta^{\prime} p_{y}^{\prime}}\right\rangle\right\rangle,
\end{aligned}
$$

where the last terms of each of (40a) and (40b) represents the divergence of three-dimensional form stress (i.e., the residual effect of pressure perturbations). Both the Coriolis term and the tendency term of (40a) and (40b) have been written in terms of the sum of the EM velocity and the Stokes-drift velocity: namely, the LM velocity. The Coriolis term of (40a) and (40b) may be interpreted as

$$
\begin{aligned}
& -f\left(\bar{v}+v^{\text {Stokes }}\right)-\beta\left(\overline{\eta^{\prime} v^{\prime}}\right) \\
& \quad=-\overline{f v}-\left(\overline{\xi^{\prime} f v^{\prime}}\right)_{x}-\left(\overline{\eta^{\prime} f v^{\prime}}\right)_{y}-\left(\overline{\zeta^{\prime} f v^{\prime}}\right)_{z} \text { and } \\
& +f\left(\bar{u}+u^{\text {Stokes }}\right)+\beta\left(\overline{\eta^{\prime} u^{\prime}}\right) \\
& =+\overline{f u}+\left(\overline{\bar{\xi}^{\prime} f u^{\prime}}\right)_{x}+\left(\overline{\eta^{\prime} f u^{\prime}}\right)_{y}+\left(\overline{\bar{\zeta}^{\prime} f u^{\prime}}\right)_{z} .
\end{aligned}
$$


Thus, the set of (40a) and (40b), combined with (41a) and (41b), represents an Eulerian approximation for the three-dimensional LM momentum equations. ${ }^{10}$

In the rest of this subsection, we specialize for, as in section $2 \mathrm{~g}$, waves that are nearly plane in the horizontal direction (such as MIGWs or MRWs; see Table 2). Using (25a)-(25d), we rewrite each component of the three-dimensional form stress in (40a) and (40b) as

$$
\begin{aligned}
& \overline{\xi^{\prime} p_{x}^{\prime}} \simeq \overline{u_{\theta}^{\prime} p_{\theta}^{\prime}} k / \sigma=\overline{u^{\prime} p^{\prime}} k / \sigma, \\
& \overline{\eta^{\prime} p_{x}^{\prime}} \simeq \overline{v_{\theta}^{\prime} p_{\theta}^{\prime}} k / \sigma=\overline{v^{\prime} p^{\prime}} k / \sigma, \\
& \overline{\zeta^{\prime} p_{x}^{\prime}} \simeq \overline{w_{\theta}^{\prime} p_{\theta}^{\prime}} k / \sigma=\overline{w^{\prime} p^{\prime}} k / \sigma, \\
& \overline{\xi^{\prime} p_{y}^{\prime}} \simeq \overline{u_{\theta}^{\prime} p_{\theta}^{\prime}} l / \sigma=\overline{u^{\prime} p^{\prime}} l / \sigma, \\
& \overline{\eta^{\prime} p_{y}^{\prime}} \simeq \overline{v_{\theta}^{\prime} p_{\theta}^{\prime}} l / \sigma=\overline{v^{\prime} p^{\prime}} l / \sigma, \text { and } \\
& \overline{\zeta^{\prime} p_{y}^{\prime}} \simeq \overline{w_{\theta}^{\prime} p_{\theta}^{\prime}} l / \sigma=\overline{w^{\prime} p^{\prime}} l / \sigma,
\end{aligned}
$$

where both $\left\langle\left\langle\xi^{\prime}, \eta^{\prime}, \zeta^{\prime}\right\rangle\right\rangle=\left\langle\left\langle-\xi_{\theta \theta}^{\prime},-\eta_{\theta \theta}^{\prime},-\zeta_{\theta \theta}^{\prime}\right\rangle\right\rangle \simeq\left\langle\left\langle u_{\theta}^{\prime}\right.\right.$, $\left.\left.v_{\theta}^{\prime}, w_{\theta}^{\prime}\right\rangle\right\rangle / \sigma$ and $\overline{(\sin \theta)^{2}}=\overline{(\cos \theta)^{2}}$ have been used. The set of (42a)-(42f) allows us to interpret the three-dimensional form stress in (40a) and (40b) as the CE flux in (26a) and (26b), which has already been suggested, for example, in (20) of Noda (2010).

To summarize, replacing the EM velocity in the Coriolis term of (5a) and (5b) with the LM velocity leads to two consequences. First, the Reynolds stress in (5a) and (5b) is replaced by the form stress, as in (40a) and (40b). Second, the tendency term of (40a) and (40b) is written in terms of the LM velocity. Both consequences are implicit in M06 and KS13, who have used analytical solutions for waves (where wave

\footnotetext{
${ }^{10}$ The tendency, Coriolis, and pressure gradient terms of (40a) and (40b) consist of quantities that may be written using the same operator $\bar{A}+\left(\overline{\bar{\xi}^{\prime} A^{\prime}}\right)_{x}+\left(\overline{\eta^{\prime} A^{\prime}}\right)_{y}+\left(\overline{\zeta^{\prime} A^{\prime}}\right)_{z}$ for $A=u, v, p_{x}, p_{y}, f v$, and $f u$ (i.e., an Eulerian approximation for the LM operator). Note that only the linear terms (i.e., the tendency, Coriolis, and pressure gradient terms) of the standard EM momentum equations (5a) and (5b) may be expressed using this operator in (40a) and (40b). The mean-flow advection term of (40a) and (40b) is out of the effect of this operator. This is because both sufficiently weak mean flows and small-amplitude linear waves have been assumed in the present study. The set of (40a) and (40b) is closely related to a type of LM momentum equation in the previous literature that has been written for the development of the LM velocity (not to be confused with another type of LM momentum equation that is written for the development of the LM velocity minus the GL pseudomomentum vector). See AM78a and AG14 (see their Table 1) for details. A related explanation appears in footnote 14.
}

amplitude, wavenumber, and wave frequency are practically constant; see footnote 4$)$.

The CE flux in (42a)-(42f) is proportional to the pressure flux $\left\langle\left\langle u^{\prime} p^{\prime}, v^{\prime} p^{\prime}, w^{\prime} p^{\prime}\right\rangle\right\rangle$ in (14), and thus is not, after application of a low-pass filter, parallel to the group velocity of MRWs (sections $2 \mathrm{~d}$ and $2 \mathrm{~g}$ ). Moreover $\sigma / k$ and $\sigma / l$ in (42a)-(42f) are not readily available from model output. For these reasons, the Eulerian approximation for the LM momentum equations (40a) and (40b) with the three-dimensional form stress are not used in the rest of this paper. Likewise, the CE pseudomomentum equations (23a) and (23b) are not used in the rest of this paper.

\section{b. Low-pass-filtered momentum equations based on the IB flux}

We shall seek a more useful expression for low-pass time-filtered momentum equations concerning the effect of waves on the mean flow. As mentioned in section $2 \mathrm{~h}$, the IB pseudomomentum vector is useful because (i) the XIB flux in (27a) is parallel to the group velocity of MRWs, (ii) the XIB and YIB fluxes in (27a) and (27b) are in an expression that is suitable for model diagnosis (i.e., $k, l, \sigma, \xi^{\prime}$, and $\eta^{\prime}$ are absent), (iii) it does not matter whether waves are nearly plane or nonplane in the horizontal direction, and (iv) there is a clear relationship [(20a) and (20b)] between the IB pseudomomentum vector and the DI wave activity. All equations shown below are applicable to the various types of linear hydrostatic neutral waves in a planetary fluid, such as MIGWs, MRWs, and EQWs.

Substitution of only the vertical component of the Reynolds stress (39c) and (39f) to the standard EM momentum equations (5a) and (5b) yields (Fig. 2 )

$$
\begin{aligned}
(\bar{u} & \left.+u^{\mathrm{qs}}\right)_{t}+\nabla \cdot(\overline{\mathbf{U}} \bar{u})-f\left(\bar{v}+v^{\mathrm{qs}}\right) \\
& =-\bar{p}_{x}-\nabla \cdot\left\langle\left\langle\overline{u^{\prime} u^{\prime}}, \overline{v^{\prime} u^{\prime}}, \overline{\zeta^{\prime} p_{x}^{\prime}}\right\rangle\right\rangle \\
& =-(\overline{p-G+K})_{x}-\nabla \cdot\left\langle\left\langle\overline{E-v^{\prime} \boldsymbol{v}^{\prime}}, \overline{v^{\prime} u^{\prime}}, \overline{\zeta^{\prime} p_{x}^{\prime}}\right\rangle\right\rangle \text { and }
\end{aligned}
$$

$$
\begin{aligned}
(\bar{v} & \left.+v^{\mathrm{qs}}\right)_{t}+\nabla \cdot(\overline{\mathbf{U}} \bar{v})+f\left(\bar{u}+u^{\mathrm{qs}}\right) \\
& =-\bar{p}_{y}-\nabla \cdot\left\langle\left\langle\overline{u^{\prime} v^{\prime}}, \overline{v^{\prime} v^{\prime}}, \overline{\zeta^{\prime} p_{y}^{\prime}}\right\rangle\right\rangle \\
& =-(\overline{p-G+K})_{y}-\nabla \cdot\left\langle\left\langle\overline{u^{\prime} v^{\prime}}, \overline{E-u^{\prime} u^{\prime}}, \overline{\zeta^{\prime} p_{y}^{\prime}}\right\rangle\right\rangle,
\end{aligned}
$$

where the last line of each of (43a) and (43b) has been written in such a way as to single out the threedimensional divergence of the IB flux in each of (27a) and (27b), respectively. It should be noted that the tendency term as well as the Coriolis term of (43a) and (43b) has been written in terms of the sum of the EM velocity and the quasi-Stokes velocity. Thus, the set of (43a) and (43b) represents an Eulerian approximation 
for the thickness-weighted isopycnal-mean momentum equations. ${ }^{11}$

The first term on the right-hand sides of (43a) and (43b) contains the quantity $\overline{-G+K}$, which is the wave kinetic energy minus the wave potential energy. To manipulate this quantity, we substitute the virial theorem (35) into (43a) and (43b) to yield (Fig. 2)

$$
\begin{aligned}
& {\left[\bar{u}+u^{\mathrm{qs}}+(\underbrace{\bar{\xi}^{\prime} u^{\prime}+\eta^{\prime} \boldsymbol{v}^{\prime}}_{\simeq 0})_{x} / 2\right]_{t}+\nabla \cdot(\overline{\mathbf{U}} \bar{u})-f\left[\bar{v}+v^{\mathrm{qs}}+\left(\overline{\bar{\xi}^{\prime} \boldsymbol{v}^{\prime}-\eta^{\prime} u^{\prime}}\right)_{x} / 2\right]} \\
& =-(\overline{p+\Lambda})_{x}-\nabla \cdot\left\langle\left\langle\overline{E-v^{\prime} v^{\prime}}, \overline{v^{\prime} u^{\prime}}, \overline{\zeta^{\prime} p_{x}^{\prime}}\right\rangle\right\rangle \quad \text { and } \\
& {\left[\bar{v}+v^{\mathrm{qs}}+(\underbrace{\overline{\xi^{\prime} u^{\prime}+\eta^{\prime} v^{\prime}}}_{\simeq 0})_{y} / 2\right]_{t}+\nabla \cdot(\overline{\mathbf{U}} \bar{v})+f\left[\bar{u}+u^{\mathrm{qs}}-\left(\overline{\xi^{\prime} v^{\prime}-\eta^{\prime} u^{\prime}}\right)_{y} / 2\right]+\beta\left(\overline{\eta^{\prime} u^{\prime}-\xi^{\prime} \boldsymbol{v}^{\prime}}\right) / 2} \\
& =-(\overline{p+\Lambda})_{y}-\nabla \cdot\left\langle\left\langle\overline{u^{\prime} v^{\prime}}, \overline{E-u^{\prime} u^{\prime}}, \overline{\zeta^{\prime} p_{y}^{\prime}}\right\rangle\right\rangle,
\end{aligned}
$$

where the quantity $\overline{\xi^{\prime} u^{\prime}+\eta^{\prime} v^{\prime}}$ in the tendency term of each equation averages to zero because of the phase relationship of neutral waves satisfying the WKB approximation. Nevertheless, this term shall be kept in what follows (thus, all equations in this section are written using an equal sign), since retaining this term will prove useful in a future study for nonneutral waves.

Equations (44a) and (44b) represent a skeleton model for the generalized TEM momentum equations that have been targeted in the atmospheric literature. Indeed, the sum of the EM velocity and the quasi-Stokes velocity, $\bar{v}+v^{\mathrm{qs}}$, in the Coriolis term of (44a) corresponds to the velocity $\boldsymbol{v}^{*}=\overline{\bar{v}}_{a}+\left(-\overline{\overline{\boldsymbol{\rho}^{\dagger} \boldsymbol{v}^{\dagger}}} / \overline{\bar{\rho}}_{z}\right)_{z}$ in the classical TEM momentum equation (3). Likewise, the meridional and vertical components of the XIB flux in (44a) correspond to $\left\langle\left\langle F^{y}, F^{z}\right\rangle\right\rangle$ in the classical TEM momentum equation (3). The set of (44a) and (44b) represents a unified

\footnotetext{
${ }^{11}$ The tendency, Coriolis, and pressure gradient terms of (43a) and (43b) consist of quantities that may be written using the same operator $\bar{A}+\left(\overline{\zeta^{\prime} A^{\prime}}\right)_{z}$ for $A=u, v, p_{x}$, and $p_{y}$ (i.e., an Eulerian approximation for the thickness-weighted-mean operator). Note that only the linear terms (i.e., the tendency, Coriolis, and pressure gradient terms) of the standard EM momentum (5a) and (5b) may be expressed using this operator in (43a) and (43b). The mean-flow advection term and the horizontal Reynolds stress term (see the second line of each equation) of (43a) and (43b) are out of the effect of this operator. This is because both sufficiently weak mean flows and small-amplitude linear waves have been assumed in the present study. The set of (43a) and (43b) is closely related with the mass-weighted isentropic-mean momentum equations in Andrews (1983a), Bleck (1985), Tung (1986), and Iwasaki (1989, 2001), as well as the thickness-weighted isopycnal-mean momentum equations in Greatbatch and McDougall (2003), Jacobson and Aiki (2006), Aiki and Richards (2008), and Young (2012).
}

expression for the effect of the various types of linear hydrostatic neutral waves in a planetary fluid, such as MIGWs, MRWs, and EQWs, on mean flows. As in (43a) and (43b), the last term on the right-hand sides of (44a) and (44b) represents the divergence of the IB flux in (27a) and (27b). Substitution of $\bar{\Lambda} \simeq 0$ and $\bar{\Lambda} \simeq-\bar{E}$ (associated with MIGWs and MRWs, respectively; see section 3c and Table 2) to the generalized TEM momentum equations (44a) and (44b) yields an equation system that is consistent with the systems of M06 and TN01, respectively. ${ }^{12}$

\section{c. Low-pass-filtered momentum equations based on the tendency of the DI wave activity}

Subtraction of the IB pseudomomentum equations (27a) and (27b) from the generalized TEM momentum equations (44a) and (44b) yields (Fig. 2)

\footnotetext{
${ }^{12}$ In the limit of a sufficiently weak mean flow, the quasigeostrophic wave activity flux associated with MRWs (that has been symbolized by $\mathbf{W})$ in TN01 corresponds to minus the XIB flux $\left\langle\left\langle E-v^{\prime} v^{\prime}, v^{\prime} u^{\prime}, \zeta^{\prime} p_{x}^{\prime}\right\rangle\right\rangle$ in the present study. Likewise, in the limit of a sufficiently weak mean flow, another flux $\mathbf{W}_{s}=-\mathbf{C}_{U} M+\mathbf{W}$ in TN01 (where $\mathbf{C}_{U}$ is the apparent phase velocity and $M$ is their quasigeostrophic wave activity associated with MRWs) corresponds to minus the combined wave-induced momentum flux $\left\langle\left\langle\Lambda+E-v^{\prime} v^{\prime}, v^{\prime} u^{\prime}, \zeta^{\prime} p_{x}^{\prime}\right\rangle\right\rangle$ on the right-hand side of the generalized TEM momentum equation in (44a) of the present study. See also (8), (31), and (45)-(51) of TN01. This indicates that $\Lambda$ in (44a) and (44b) of the present study is closely related with $\mathbf{C}_{U} M$ in TN01 in the limit of a sufficiently weak mean flow. Indeed, after application of an Eulerian time average (and also in the limit of a sufficiently weak mean flow), $M$ in (26) of TN01 reduces to $\bar{M} \simeq \bar{E} /\left(|\mathbf{U}|-C_{p}\right) \simeq-\bar{E} / C_{p}$, where the symbols $\mathbf{U}$ and $C_{p}$ are adapted from TN01. Thus, $\mathbf{C}_{U} \bar{M} \simeq-\bar{E} \mathbf{C}_{U} / C_{p}=-\bar{E} \mathbf{U} /|\mathbf{U}|$, which corresponds to $\langle\langle\bar{\Lambda}, 0,0\rangle\rangle \simeq\langle\langle-\bar{E}, 0,0\rangle\rangle$ in the present study.
} 


$$
\begin{gathered}
{[\bar{u}+\underbrace{u^{\mathrm{qs}}-\overbrace{\left(\overline{\zeta_{z}^{\prime} u^{\prime}}+\overline{q^{\prime} \eta^{\prime}} / 2\right)}^{\text {IB pseudomomentum }}}_{\text {DI wave activity }}+\underbrace{\left.\left(\overline{\xi^{\prime} u^{\prime}+\eta^{\prime} v^{\prime}}\right)_{x} / 2\right]_{t}+\nabla \cdot(\overline{\mathbf{U}} \bar{u})-f\left[\bar{v}+v^{\mathrm{qs}}+\left(\overline{\bar{\xi}^{\prime} v^{\prime}-\eta^{\prime} u^{\prime}}\right)_{x} / 2\right]=-(\overline{p+\Lambda})_{x} \quad \text { and }}_{\simeq 0}} \\
{[\bar{v}+\underbrace{v^{\mathrm{qs}}-\overbrace{\left(\overline{\zeta_{z}^{\prime} v^{\prime}}-\overline{q^{\prime} \xi^{\prime}} / 2\right)}^{\text {IB pseudomomentum }}}_{\text {DI wave activity }}+(\underbrace{\overline{\xi^{\prime} u^{\prime}+\eta^{\prime} v^{\prime}}}_{\simeq 0})_{y} / 2]_{t}+\nabla \cdot(\overline{\mathbf{U}} \bar{v})+f\left[\bar{u}+u^{\mathrm{qs}}-\left(\overline{\xi^{\prime} v^{\prime}-\eta^{\prime} u^{\prime}}\right)_{y} / 2\right]=-(\overline{p+\Lambda})_{y},}
\end{gathered}
$$

where $f=f_{0}+\beta y$ is understood. A nice feature is that the extra $\beta$ term in the meridional component of the generalized TEM momentum equation (44b) has been cancelled out in (45b). It is interesting that, although the meridional component of the IB pseudomomentum vector is not conserved, (45a) and (45b) are (apparently) symmetric in the zonal and meridional directions.

Equations (45a) and (45b) represent a skeleton model for the generalized MEM momentum equations [see (4)]. Indeed, the tendency terms of (45a) and (45b) have been written in terms of the EM velocity plus the quasi-Stokes velocity minus the IB pseudomomentum [and also the horizontal gradient of $\left(\overline{\xi^{\prime} u^{\prime}+\eta^{\prime} v^{\prime}}\right) / 2$, which is nearly zero because of the phase relationship of neutral waves satisfying the WKB approximation], which may be interpreted as the sum of the EM velocity and the DI wave activity as in (20a) and (20b). Because we have kept the tendency of the quasi-Stokes velocity in (43a) and (43b), we were able to derive the DI wave activity. To summarize, the generalized MEM momentum equations (45a) and (45b) have been written for the development of the sum of the EM velocity and the DI wave activity and thus represent a generalized expression for (4), which is related with the nonacceleration theorem. It should be also noted that both the generalized TEM momentum equations (44a) and (44b) and the generalized MEM momentum equations (45a) and (45b) involve the horizontal gradient of $\bar{\Lambda}$, which has been little mentioned in previous studies.

How can one use the generalized MEM equations (45a) and (45b) in a future study? For example, inclusion of turbulent viscosity terms to the perturbation momentum equations (6a) and (6b) will determine, via the associated modification of the perturbation EPV equation (10), the form of the viscosity term in the IB pseudomomentum equations (27a) and (27b), as in Haynes (1988). Likewise, inclusion of the turbulent viscosity terms in (6a) and (6b) will determine, via the associated modification of (35), (39c), and (39f), the form of the viscosity term in the generalized TEM momentum equations (44a) and (44b). Merging the modified versions of the IB pseudomomentum equations (27a) and (27b) and the TEM momentum equations (44a) and (44b) will determine the form of the turbulent viscosity term in the MEM momentum equations (45a) and (45b). As noted by AG14, who have performed a corresponding analysis (for oceanic surface gravity waves using a variant of the generalized LM theory), the turbulent viscosity term of the MEM momentum equations will not be written in a flux divergence form. There should be a term representing the transfer of momentum from waves and the mean flow associated with the dissipation of wave energy. The supplemental material of the present study will be useful for a future study to follow the procedure of this paragraph.

\section{Interrelationships with different three-dimensional theories}

Here we investigate how quantities relevant to $\bar{\Lambda}$ have been hidden in the isopycnal-mean theory (section 5a) and the generalized LM theory (section 5b). Herein, we will refer to thickness-weighted isopycnal mean (WIM) and unweighted isopycnal mean (UIM).

\section{a. Origin of the quantity $K-G$}

The presence of $\Lambda$ in both the generalized TEM and MEM momentum equations in (44) and (45) is attributed to the use of (35) in section $4 \mathrm{~b}$ to manipulate $K-G$ in the WIM momentum equations (43a) and (43b), which may be traced back to the IB pseudomomentum equations (27a) and (27b). Below, we explain why these equations contain $K-G$.

Subtraction of the generalized Taylor-Bretherton identity (28a) and (28b) from the WIM momentum equations (43a) and (43b) yields (Fig. 2)

$$
\begin{aligned}
& \underbrace{\left(\bar{u}+\overline{\zeta^{\prime} u_{z}^{\prime}}\right)_{t}}_{\tilde{u}}+\nabla \cdot(\overline{\mathbf{U}} \bar{u})-[f \underbrace{\left(\bar{v}+\overline{\zeta^{\prime} v_{z}^{\prime}}\right)}_{\tilde{v}}+\overline{\left(v_{x}^{\prime}-u_{y}^{\prime}\right) v^{\prime}}] \\
& =-(\underbrace{\bar{p}-\bar{G}}_{\tilde{p}}+\bar{K})_{x} \text { and }
\end{aligned}
$$




$$
\begin{aligned}
& (\underbrace{\bar{v}+\overline{\zeta^{\prime} v_{z}^{\prime}}}_{\tilde{v}})_{t}+\nabla \cdot(\overline{\mathbf{U}} \bar{v})+[f \underbrace{\left(\bar{u}+\overline{\zeta^{\prime} u_{z}^{\prime}}\right)}_{\tilde{u}}+\overline{\left(v_{x}^{\prime}-u_{y}^{\prime}\right) u^{\prime}}] \\
& =-(\underbrace{\bar{p}-\bar{G}}_{\tilde{p}}+\bar{K})_{y},
\end{aligned}
$$

which has been derived using $q^{\prime}=v_{x}^{\prime}-u_{y}^{\prime}-f \zeta_{z}^{\prime}$. Equation (46a) contains $-\overline{\left(v_{x}^{\prime}-u_{y}^{\prime}\right) v^{\prime}}$ on the left-hand side and $-\bar{K}_{x}$ on the right-hand side, which is a vectorinvariant form of the horizontal advection term $\overline{u^{\prime} u_{x}^{\prime}+v^{\prime} u_{y}^{\prime}}$. Likewise, $(46 \mathrm{~b})$ contains $+\overline{\left(v_{x}^{\prime}-u_{y}^{\prime}\right) u^{\prime}}$ on the left-hand side and $-\bar{K}_{y}$ on the right-hand side, which is a vector-invariant form of the horizontal advection term $\overline{u^{\prime} \boldsymbol{v}_{x}^{\prime}+\boldsymbol{v}^{\prime} \boldsymbol{v}_{y}^{\prime}}$. This explains why $\bar{K}$ has the same status as $\bar{p}$. However, it is still unclear why the sign in front of $\bar{G}$ is negative in (46a) and (46b), a topic we shall discuss in what follows.

We seek to understand the tendency, the Coriolis, and the pressure gradient terms of (46a) and (46b) using an approximate expression for the UIM (denoted by the tilde) of an arbitrary quantity $A$, as follows:

$$
\begin{aligned}
\tilde{A} & \equiv \bar{A}+\overline{\zeta^{\prime} A_{z}}+\overline{\left(\zeta^{\prime 2} / 2\right) A_{z z}}+\cdots \\
& =\bar{A}+\overline{\zeta^{\prime} A_{z}^{\prime}}+\overline{\left(\overline{\zeta^{\prime 2}} / 2\right) \bar{A}_{z z}+\cdots}
\end{aligned}
$$

which has been written up to the second order in terms of a Taylor expansion in the vertical direction; the second line omits the triple and higher product of perturbation quantities. The right-hand side of (47) has been written in terms of quantities averaged in Eulerian coordinates. Equation (47) allows us to interpret both the tendency term and the Coriolis term of (46a) and (46b) in terms of $\tilde{u}$ and $\tilde{v}$. An exception is that the second-order derivative term on the last line of (47) (viz., $\bar{A}_{z z}=\bar{u}_{z z}$ and $\bar{v}_{z z}$ ) is absent in (46a) and (46b). This is because the IB pseudomomentum equations (27a) and (27b), as well as the generalized TaylorBretherton identity (28a) and (28b), have been derived from the equation system (6a)-(11) for linear waves with no mean flow. On the other hand, substitution of $A=p$ to (47) yields

$$
\begin{aligned}
\tilde{p} & =\bar{p}+\overline{\zeta^{\prime} p_{z}^{\prime}}+\left(\overline{\zeta^{12}} / 2\right) \bar{p}_{z z}+\cdots \\
& =\bar{p}-\overline{\zeta^{12}} N^{2}+\left(\overline{\zeta^{12}} / 2\right) N^{2}+\cdots \\
& =\bar{p}-\underbrace{\left.\overline{\left(\zeta^{12}\right.} / 2\right) N^{2}}_{\bar{G}}+\cdots
\end{aligned}
$$

where both (9) and $N^{2} \equiv-\bar{\rho}_{z} g / \rho_{0}$ (i.e., hydrostatic relation for the wave and mean fields, respectively) have (a) Eulerian mean pressure

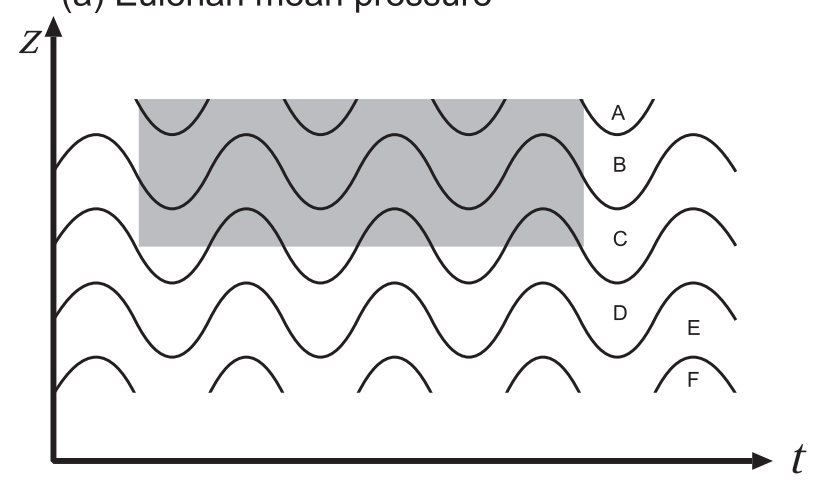

(b) Isopycnal mean pressure

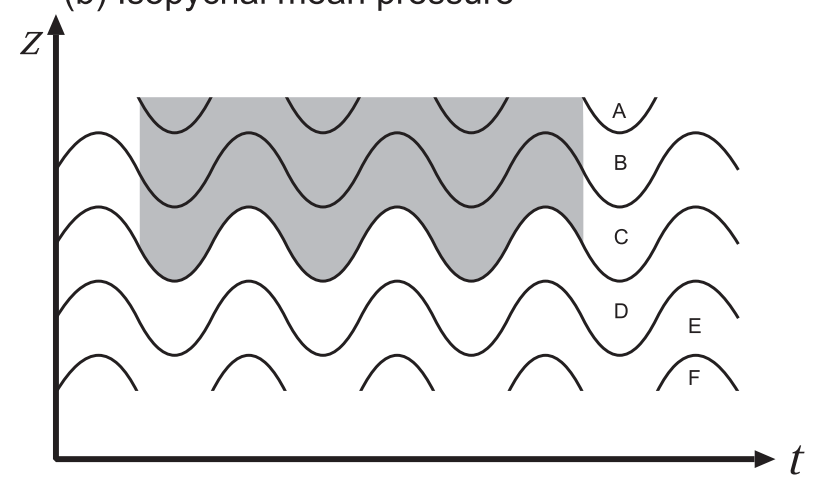

FIG. 3. A time series for the vertical profile of density in a continuously stratified fluid (contour). The shading shows the area of density that is used to calculate (a) the Eulerian-mean hydrostatic pressure and (b) the isopycnal-mean hydrostatic pressure. The density of fluid particles in regions A-F increases in an alphabetical order. Fluid particles in region $\mathrm{D}$ are counted in the Eulerian-mean pressure but not the isopycnal-mean pressure, which explains why the Eulerian-mean pressure is always greater than the isopycnal-mean pressure compared at the same reference height.

been used to derive the second line, and the last line indicates that the EM pressure $\bar{p}$ is always greater than the UIM pressure $\tilde{p}$ on the left-hand side. The difference between $\bar{p}$ and $\tilde{p}$ is illustrated in Fig. 3, where the density of fluid particles in regions A-F increases in an alphabetical order. The EM pressure partially includes particles in the region D (of greater density) and also partially excludes particles in the region $\mathrm{C}$ (of smaller density). The gain of mass for $\bar{p}$ from the region $\mathrm{D}$ is greater than the loss of mass for $\bar{p}$ from the region $\mathrm{C}$, which indicates that $\bar{p}$ is always greater than $\tilde{p}$ compared at a given reference height and is consistent with (48). The above interpretation is also consistent with a generalized expression for the wave potential energy: $\int_{z}^{z+\zeta^{\prime}} \rho d z g / \rho_{0}$, as given by Holliday and McIntyre (1981). 
Substitution of $A=p_{x}$ into (47) yields $\widetilde{\left(p_{x}\right)}=\overline{\left(p_{x}\right)}+$ $\overline{\zeta^{\prime} p_{x z}^{\prime}}=\overline{\left(p_{x}\right)}-\overline{\zeta^{\prime} \zeta_{x}^{\prime}} N^{2}=\partial_{x}(\bar{p}-\bar{G})=\partial_{x}(\tilde{p})$, where both the subscript $x$ and the symbol $\partial_{x}$ refer to the same zonal gradient operator at fixed height. Namely, concerning the treatment of hydrostatic pressure, the UIM operator and the fixed-height zonal gradient operator commute. This can be also confirmed without using the Taylor expansion in (47) but using density coordinates (to be explained in detail in our future study). The result is that the pressure gradient term of (46a) may be interpreted as $\partial_{x}(\bar{p}-\bar{G})=\partial_{x}(\tilde{p})=\widetilde{\left(p_{x}\right)}$. The same analysis holds for the pressure gradient term in (46b). Thus, the set of (46a) and (46b) represents an Eulerian approximation for the UIM momentum equations in a vector-invariant form. ${ }^{13}$ This explains why the sign in front of $\bar{G}$ in (46a) and (46b) is opposite to the sign in front of $\bar{K}$. To summarize, the generalized Taylor-Bretherton identity (28a) and (28b) may be interpreted as the difference of the WIM momentum equations (43a) and (43b) and the UIM momentum equations (46a) and (46b) in a vector-invariant form. This explains the origin of $-\bar{K}+\bar{G}$ in (28a) and (28b) and thus in the IB pseudomomentum equations (27a) and (27b).

Additional results may be obtained if the UIM velocity is interpreted as the sum of the EM velocity and the gravity wave part of the DI wave-activity vector in (20a) and (20b): $\langle\langle\tilde{u}, \tilde{v}\rangle\rangle=\left\langle\left\langle\bar{u}+\overline{\zeta^{\prime} u_{z}^{\prime}}, \bar{v}+\overline{\zeta^{\prime} v_{z}^{\prime}}\right\rangle\right\rangle$. This indicates that the UIM momentum equations (46a) and (46b) are closely related with the MEM momentum equations (45a) and (45b), concerning the treatment of hydrostatic gravity waves. Without the above recognitions (one is associated with the Taylor-Bretherton identity, mentioned in the previous paragraph, and one is associated with the gravity wave part of the wave activity, mentioned in the present paragraph), previous studies have not paid a significant attention to the UIM momentum equations (46a) and (46b), except for Tung (1986) and

\footnotetext{
${ }^{13}$ The tendency, Coriolis, and pressure gradient terms of (46a) and (46b) consist of quantities that may be written using the same operator [(47)] for $A=u, v$, and $p$ (i.e., an Eulerian approximation for the unweighted isopycnal-mean operator). Note that only the linear terms (i.e., the tendency, Coriolis, and pressure gradient terms) of the standard EM momentum equations (5a) and (5b) may be expressed using this operator in (46a) and (46b). The mean-flow advection term and the term in a vector-invariant form of (46a) and (46b) are out of the effect of this operator. This is because both sufficiently weak mean flows and small-amplitude linear waves have been assumed in the present study. The set of (43a) and (43b) is closely related with the unweighted isopycnal-mean momentum equations in Tung (1986) and Greatbatch (1998).
}

Greatbatch (1998). Probably the UIM velocity has been thought not to be a robust quantity because its volume integral does not become identical to that of the EM velocity. It is the WIM velocity for which the volume integral becomes identical to that of the EM velocity [see the pileup rule in Aiki and Yamagata (2006)]. See also Fig. 2 to note that what is missing in both WIM and UIM is the virial theorem (35), which is why $\bar{\Lambda}$ is absent in the WIM and UIM momentum equations.

\section{b. Prototype of the quantity $\bar{p}+\bar{\Lambda}$}

The right-hand side of the MEM momentum equations (45a) and (45b) has been written as minus the horizontal gradient of $\bar{p}+\bar{\Lambda}$, where $\bar{p}$ is the EM of hydrostatic pressure. As shown below, the transformed expression of the LM (TLM) ${ }^{14}$ momentum equations of AM78a contains a set of terms that may be interpreted as the prototype of $\bar{p}+\bar{\Lambda}$.

Equations (45a) and (45b) have been written for the development of the sum of the EM velocity and the DI wave-activity vector. Each component of the DI waveactivity vector may be expanded as

$$
\begin{aligned}
\underbrace{\overline{\zeta^{\prime} u_{z}^{\prime}}-\overline{\eta^{\prime} q^{\prime} / 2}=}_{\text {DI wave activity }} & -\left(\overline{\xi^{\prime} u^{\prime}+\eta^{\prime} v^{\prime}}\right)_{x}-\left(f \overline{\xi^{\prime} \eta^{\prime}}\right)_{x} / 2-\left(\overline{f \eta^{\prime 2}}\right)_{y} / 2 \\
& +\underbrace{\left(\overline{\bar{\xi}^{\prime} u_{x}^{\prime}+\eta^{\prime} u_{y}^{\prime}+\zeta^{\prime} u_{z}^{\prime}}\right)}_{u^{\text {Stokes }}} \\
& -\underbrace{\left.\overline{\left[-u^{\prime} \xi_{x}^{\prime}-v^{\prime} \eta_{x}^{\prime}+f\left(\xi_{x}^{\prime} \eta^{\prime}-\xi^{\prime} \eta_{x}^{\prime}\right) / 2\right.}\right]}_{\text {GL pseudomomentum }} \text { and }
\end{aligned}
$$

\footnotetext{
${ }^{14}$ In general, the LM framework allows for low-pass-filtered momentum equations to be written in two separate expressions (Lagrange 1788), referred to as the direct and transformed expressions in AG14. AM78a have shown that (i) the direct expression of the LM momentum equations is written for the development of the three-dimensional LM velocity [see (8.7a) of AM78a], which corresponds to (40a) and (40b) in the present study, and (ii) the transformed expression of the LM momentum equations is written for the development of the three-dimensional LM velocity minus the GL pseudomomentum vector [see (3.8) of AM78a], which corresponds to (51a) and (51b) in the present study. The transformed expression of the LM momentum equations has been widely used in the literature of oceanic surface gravity waves to rederive the Craik and Leibovich (1976) vortex force and to explain the transfer of momentum from waves to circulation associated with the dissipation of waves. See AG14 and references therein.
} 


$$
\begin{aligned}
\underbrace{\overline{\zeta^{\prime} v_{z}^{\prime}}+\overline{\xi^{\prime} q^{\prime}} / 2}_{\text {DI wave activity }}= & -\left(\overline{\xi^{\prime} u^{\prime}+\eta^{\prime} v^{\prime}}\right)_{y}+\left(f \overline{\xi^{\prime 2}}\right)_{x} / 2+\left(f \overline{\bar{\xi}^{\prime} \eta^{\prime}}\right)_{y} / 2 \\
& +\underbrace{\left(\overline{\xi^{\prime} v_{x}^{\prime}+\eta^{\prime} v_{y}^{\prime}+\zeta^{\prime} v_{z}^{\prime}}\right)}_{v^{\text {Stokes }}} \\
& -\underbrace{\left[\overline{-u^{\prime} \xi_{y}^{\prime}-v^{\prime} \eta_{y}^{\prime}+f\left(\xi_{y}^{\prime} \eta^{\prime}-\xi^{\prime} \eta_{y}^{\prime}\right) / 2}\right]}_{\text {GL pseudomomentum }},
\end{aligned}
$$

where (11) and $q^{\prime} \equiv v_{x}^{\prime}-u_{y}^{\prime}-f \zeta_{z}^{\prime}$ have been used, and $u^{\text {Stokes }}$ and $v^{\text {Stokes }}$ on the last lines have been defined in (12a) and (12b) (detailed derivation in the supplemental material). The last term of each equation may be interpreted as an Eulerian approximation for the GL pseudomomentum in (3.1) of AM78a.

We now expand $\bar{p}+\bar{\Lambda}$ on the right-hand sides of (45a) and (45b):

$$
\begin{aligned}
& \{\bar{u}+u^{\text {Stokes }}-\underbrace{\left[\overline{-u^{\prime} \xi_{x}^{\prime}-v^{\prime} \eta_{x}^{\prime}+f\left(\xi_{x}^{\prime} \eta^{\prime}-\xi^{\prime} \eta_{x}^{\prime}\right) / 2}\right]}_{\text {GL pseudomomentum }}\}_{t}+\nabla \\
& =-[\underbrace{\left(\tilde{p}+\overline{\xi^{\prime} p_{x}^{\prime}}+\overline{\eta^{\prime} p_{y}^{\prime}}\right)}_{\text {LM pressure }} \underbrace{-\bar{K}-f\left(\overline{\xi^{\prime} v^{\prime}-\eta^{\prime} u^{\prime}}\right) / 2}_{\text {Bernoulli head }}]_{x} \text { and } \\
& \{\bar{v}+v^{\text {Stokes }}-\underbrace{\left[\overline{-u^{\prime} \xi_{y}^{\prime}-v^{\prime} \eta_{y}^{\prime}+f\left(\xi_{y}^{\prime} \eta^{\prime}-\xi^{\prime} \eta_{y}^{\prime}\right) / 2}\right]}_{\text {GL pseudomomentum }}\}_{t}+\nabla \cdot \\
& =-[\underbrace{\left(\tilde{p}+\overline{\xi^{\prime} p_{x}^{\prime}}+\overline{\eta^{\prime} p_{y}^{\prime}}\right)}_{\text {LM pressure }} \underbrace{-\bar{K}-f\left(\overline{\bar{\xi}^{\prime} v^{\prime}-\eta^{\prime} u^{\prime}}\right) / 2}_{\text {Bernoulli head }}]_{y} \text {, }
\end{aligned}
$$$$
(\overline{\mathbf{U}} \bar{u})-f\left(\bar{v}+v^{\text {Stokes }}\right)-\beta\left(\overline{\eta^{\prime} v^{\prime}}\right)
$$
yields

where the second line has been derived using (9) and (35), and the last line has been derived using (48). The first term on the last line of (50) represents an Eulerian approximation for the three-dimensional LM of hydrostatic pressure. Substitution of (49a)-(50) to the MEM momentum equations (45a) and (45b)

which represent an Eulerian approximation for the TLM momentum equations of AM78a. Equations (51a) and (51b) are consistent with (3.8) of AM78a (except that the planetary $\beta$ effect is implicit in the original paper by AM78a). Indeed, (51a) and (51b) have been written for the development of the threedimensional LM velocity minus the GL pseudomomentum vector. A known feature for the TLM momentum equations of AM78a is that the horizontal gradient term on the right-hand sides of (51a) and (51b) contain $-\bar{K}$, in contrast to $+\bar{K}$ in the vector-invariant form of momentum equations (46a) and (46b). The quantity $-\bar{K}-f\left(\overline{\bar{\xi}^{\prime} \boldsymbol{v}^{\prime}-\eta^{\prime} u^{\prime}}\right) / 2$ in (51a) and (51b) has sometimes been referred to as the Bernoulli head. In terms of both characterization (depending on MIGWs, MRWs, and EQWs) and physical interpretation, understanding of the LM pressure and the Bernoulli head has been thought to be formidable in previous studies for the generalized LM theory of AM78a. Equation (50) herein allows us to replace the problem through our understanding of $\bar{p}+\bar{\Lambda}$. We have already investigated the characteristics of $\bar{\Lambda}$ for MIGWs, MRWs, and EQWs and have also explained how to estimate $\bar{\Lambda}$ analytically and numerically (sections $3 \mathrm{~b}$ and $3 c$ ).

The TLM equations (51a) and (51b) have some extra $\beta$ terms, whereas the MEM equations (45a) and (45b) have no extra $\beta$ term. Furthermore the MEM equations have been written for the development of the sum of the EM velocity and the DI wave-activity vector. These features of MEM are more appropriate for a model diagnosis than the TLM momentum equations (51a) 
and (51b). Since MEM represents the compilation of all four aspects of the Eliassen-Palm theory (section 1), future studies should develop advanced versions of (45a) and (45b) to account for finite-amplitude waves in a sheared mean flow and also for the dissipation of wave energy, which will be relevant to parallel progress in the study of oceanic surface gravity waves (see footnote 14).

\section{Summary}

Classical wave-mean flow interaction theory concerning the Eliassen-Palm relation is extended in the present study to allow for a unified treatment of midlatitude inertia-gravity waves (MIGWs), midlatitude Rossby waves (MRWs), and equatorial waves (EQWs). In the present study, the form of what we call the impulse-bolus (IB) pseudomomentum vector has been determined from the explicit relationship (18a) and (18b) with the classical energy-based (CE) pseudomomentum vector (section $2 \mathrm{e}$ ), prior to examining conservation properties. This approach, toward a threedimensional formulation for waves at all latitudes, is similar in part to the definition of the generalized Lagrangian (GL) pseudomomentum vector in AM78a. Given the cost to handle all MIGWs, MRWs, and EQWs in a three-dimensional framework, the present study has adopted

(i) An Eulerian time-mean (EM) framework for small-amplitude waves in a hydrostatic Boussinesq fluid with a sufficiently weak mean flow. See also footnote 1 .

(ii) An approach to extend the basic result of Ripa (1982), Andrews (1983a), and Haynes (1988), who have derived the ageostrophic version of the Eliassen-Palm relation [see (2)] using a layer model or an isentropic coordinate system, to account for the other three aspects of the Eliassen-Palm theory (i.e., the Taylor-Bretherton identity and the TEM and MEM momentum equations).

(iii) An approach to derive prognostic equations for the pseudomomentum as instantaneous expressions without using either the set of the wave action and crest equations or an analytical solution for a given type of wave (sections $2 \mathrm{~g}$ and $2 \mathrm{~h}$; see also footnote 4).

For MIGWs, both the CE and IB fluxes are parallel to the group velocity of waves. For MRWs, only the XIB flux is parallel to the group velocity of waves. For EQWs propagating in the zonal direction, the meridional components of both the XCE and XIB fluxes vanish. In addition, the XIB and YIB fluxes in (27a) and (27b) are in an expression that is suitable for model diagnosis (i.e., $k, l, \sigma, \xi^{\prime}$, and $\eta^{\prime}$ are absent).

In section 3 , we have investigated what makes the three-dimensional fluxes in the CE and IB pseudomomentum equations look in different directions. Since the CE and IB pseudomomenta only differ by divergence of a vector [see (18a) and (18b)], their prognostic equations are related through the gauge transformation associated with the divergence-form wave-induced pressure $\Lambda$, which is the most important result of the present study (section 3a). Then we have investigated the characteristics of $\Lambda$ for MIGWs, MRWs, and EQWs, with two approaches for estimating $\Lambda$. The first approach is based on the combination of the virial theorem and the potential vorticity equation and is applicable to both MRWs and EQWs (section 3b). The second approach is based on the assumption of nearly plane waves in the horizontal direction and is applicable to both MIGWs and MRWs (section 3c). We have used the CE flux as a reference for affirming the direction of the IB flux, because the CE flux is proportional to (not only the pressure flux in the wave energy equation but also) the threedimensional form stress in the three-dimensional LM momentum equations (see DLM below). Namely, the three-dimensional form stress is parallel to the direction of the group velocity of MIGWs, but not for MRWs.

As illustrated in Fig. 2, we have compared the different versions of low-pass time-filtered momentum equations that have been suggested in the literature (in either a prototype form or an advanced form) and also derived some of the following in the present study:

- SEM: the standard EM momentum equations (5a) and (5b) with the divergence of the three-dimensional Reynolds stress

- DLM: (an Eulerian approximation for the direct expression of) the three-dimensional LM momentum equations (40a) and (40b) with the divergence of the three-dimensional form stress (which is parallel to the CE flux)

- WIM: (an Eulerian approximation for) the thickness-weighted isopycnal-mean momentum equations (43a) and (43b) with the divergence of the horizontal Reynolds stress and the vertical form stress

- TEM: the generalized TEM momentum equations (44a) and (44b) with the horizontal gradient of $\bar{\Lambda}$ and the divergence of the IB flux 
- MEM: the merged form of the EM momentum equations (45a) and (45b) with the horizontal gradient of $\bar{\Lambda}$

- UIM: (an Eulerian approximation for) the unweighted isopycnal-mean momentum equations (46a) and (46b) in a vector-invariant form

- TLM: (an Eulerian approximation for the transformed expression of) the three-dimensional LM momentum equations (51a) and (51b) with the horizontal gradient of the prototype of $\bar{\Lambda}$.

Expressions for the tendency term and the Coriolis term of each equation system vary accordingly (sections 4 and 5). Note that TEM, MEM, and TLM involve the horizontal gradient of $\bar{\Lambda}$, which has been little mentioned in previous studies. The TEM and MEM momentum equations of the present study are applicable to waves at all latitudes, because they have been derived from the IB pseudomomentum equations (27a) and (27b).

As illustrated in Fig. 2, the IB pseudomomentum equations (27a) and (27b) represent the difference between TEM and MEM. We have shown that the generalized Taylor-Bretherton identity (28a) and (28b) represents the difference between the WIM and UIM. Overall, what is missing in the previous isopycnal-mean theory (WIM and UIM) as compared to the generalized Eliassen-Palm theory (TEM and MEM) is the virial theorem (35), which is why $\bar{\Lambda}$ is absent in WIM and UIM. The virial theorem (35) has also allowed, via (50), for MEM to be linked to TLM. TLM contains the so-called Bernoulli head, the understanding of which has been thought to be formidable in the previous literature. We have shown that the sum of the LM pressure and the Bernoulli head in TLM becomes identical to $\bar{p}+\bar{\Lambda}$. The above relationship provides a basis for a future study aimed at achieving a unified treatment of the effect of the various types of finite-amplitude waves on a sheared mean flow and with the effect of turbulent viscosity. Key equations, such as (18a), (18b), (35), (37), and (50) and diagrams (Figs. 1 and 2 ) in the present study will be useful for understanding the interrelationships between different formulations in recent studies (TN01; M06; Nakamura and Solomon 2011; Solomon and Nakamura 2012; KS13; Maddison and Marshall 2013; Methven 2013).

To summarize, the present study has shown both the various interesting characteristics of $\bar{\Lambda}$ (for MIGWs, MRWs, and EQWs) and the importance of $\bar{\Lambda}$ in the three-dimensional version of the major formulations for the effect of waves on the mean flow (see TEM, MEM, and TLM in the above list). These abundant results, previously unmentioned, suggest a future study dedicated to an independent investigation for $\bar{\Lambda}$ in terms of, for example, a detailed physical interpretation and Hamiltonian dynamics. It is challenging, but it will be worth giving an effective name for $\bar{\Lambda}$, as discussed in appendix $\mathrm{C}$.

Acknowledgments. The authors thank Prof. Noboru Nakamura and an anonymous reviewer for a series of constructive comments to improve the manuscript. HA thanks Emeritus Professors Toshio Yamagata and Taroh Matsuno for interesting discussions. RJG is grateful for continuing support from GEOMAR.

\section{APPENDIX A}

\section{An Alternative Expression for the Wave Energy Associated with MIGWs}

In this appendix, we assume that the Coriolis parameter $f$ is a uniform constant (i.e., $f=f_{0}$ and $\beta=0$ ) and the buoyancy frequency $N$ is a uniform constant (in particular, with no variation in the vertical direction). We shall show that the quantity $\left(\overline{u_{t}^{\prime} v^{\prime}-u^{\prime} v_{t}^{\prime}}\right) /(2 f)$ on the right-hand side of (38) is equal to the wave energy associated with MIGWs.

We consider a Fourier integral in the form

$$
\begin{aligned}
p^{\prime}(x, y, z, t) & =\int P(k, l, m, \theta) d^{3} k, \\
u^{\prime}(x, y, z, t) & =\int U(k, l, m, \theta) d^{3} k, \\
v^{\prime}(x, y, z, t) & =\int V(k, l, m, \theta) d^{3} k, \\
w^{\prime}(x, y, z, t) & =\int W(k, l, m, \theta) d^{3} k, \quad \text { and } \\
\zeta^{\prime}(x, y, z, t) & =\int Z(k, l, m, \theta) d^{3} k,
\end{aligned}
$$

where $P, U, V, W$, and $Z$ are the Fourier constituents associated with $p^{\prime}, u^{\prime}, v^{\prime}, w^{\prime}$ and $\zeta^{\prime}$, respectively; $(k, l)$ and $m$ are the horizontal and vertical wavenumbers; and $\theta=k x+l y+m z-\sigma t$ is the wave phase, with $\sigma$ being the wave frequency. The Fourier integral is 
$\int d^{3} k \equiv \iiint d k d l d m$. An analytical solution for the equation system (6a)-(6e) may be written as

$$
\begin{aligned}
P & \equiv \mathcal{A} \cos \theta, \\
U & \equiv\left(P \sigma k+P_{\theta} f l\right) /\left(\sigma^{2}-f^{2}\right), \\
V & \equiv\left(-P_{\theta} f k+P \sigma l\right) /\left(\sigma^{2}-f^{2}\right), \\
W & \equiv-P \sigma m / N^{2}, \quad \text { and } \\
Z & \equiv-P_{\theta} m / N^{2},
\end{aligned}
$$

where $\mathcal{A}=\mathcal{A}(k, l, m)$ is wave amplitude for each Fourier constituent, and $P_{\theta}=-\mathcal{A} \sin \theta$ is understood (Gill 1982; Holton 1992). A dispersion relation for MIGWs is written as

$$
\frac{k^{2}+l^{2}}{\sigma^{2}-f^{2}}=\frac{m^{2}}{N^{2}}
$$

Using the analytical solution (A2a)-(A2e), we calculate the time average of the wave energy associated with MIGWs with a general spectrum:

$$
\begin{aligned}
\bar{E} & =(1 / 2) \int \overline{\left[U^{2}+V^{2}+N^{2} Z^{2}\right]} d^{3} k \\
& =(1 / 2) \int\left[\left(\overline{P^{2}} \sigma^{2}+\overline{P_{\theta}^{2}} f^{2}\right)\left(k^{2}+l^{2}\right) /\left(\sigma^{2}-f^{2}\right)^{2}+\overline{P_{\theta}^{2}} m^{2} / N^{2}\right] d^{3} k \\
& =(1 / 2) \int\left[\left(\overline{P^{2}} \sigma^{2}+\overline{P_{\theta}^{2}} f^{2}\right)+\overline{P_{\theta}^{2}}\left(\sigma^{2}-f^{2}\right)\right] m^{2} /\left[N^{2}\left(\sigma^{2}-f^{2}\right)\right] d^{3} k=\int \overline{P^{2}} \sigma^{2} m^{2} /\left[N^{2}\left(\sigma^{2}-f^{2}\right)\right] d^{3} k,
\end{aligned}
$$

where both $\overline{P P_{\theta}}=-\mathcal{A}^{2} \overline{\cos \theta \sin \theta}=0$ and $\overline{P^{2}}=$ $\mathcal{A}^{2} \overline{\cos ^{2} \theta}=\mathcal{A}^{2} \frac{\overline{\sin ^{2} \theta}}{\sin _{\theta}^{2}}$ have been used, and the third line of (A4) has been derived using the dispersion relation (A3).

On the other hand, substitution of the analytical solution (A2a)-(A2e) to the quantity $\left(\overline{u_{t}^{\prime} v^{\prime}-u^{\prime} v_{t}^{\prime}}\right) /(2 f)$ on the right-hand side of (38) yields

$$
\begin{aligned}
& \left(u_{t}^{\prime} v^{\prime}-u^{\prime} v_{t}^{\prime}\right) /(2 f) \\
& =(1 / 2) \int\left(P^{2}+P_{\theta}^{2}\right) \sigma^{2}\left(k^{2}+l^{2}\right) /\left(\sigma^{2}-f^{2}\right)^{2} d^{3} k \\
& =(1 / 2) \int\left(P^{2}+P_{\theta}^{2}\right) \sigma^{2} m^{2} /\left[N^{2}\left(\sigma^{2}-f^{2}\right)\right] d^{3} k,
\end{aligned}
$$

which has been written in an instantaneous expression, and the second line has been derived using the dispersion relation (A3). Comparing the last line of each of (A4) and (A5), one can easily see that the time average of (A5) becomes equal to the wave energy in (A4), with the understanding that $(1 / 2)\left(\overline{P^{2}+P_{\theta}^{2}}\right)=\overline{P^{2}}$ [i.e., $(1 / 2)\left(\overline{\cos ^{2} \theta+\sin ^{2} \theta}\right)=\overline{\cos ^{2} \theta}$. We conclude that $\left(\overline{u_{t}^{\prime} v^{\prime}-u^{\prime} v_{t}^{\prime}}\right) /(2 f)=\bar{E}$ for MIGWs with a general spectrum. This result applies only to MIGWs, not to MRWs or EQWs. Additional features of the quantity $\left(u_{t}^{\prime} v^{\prime}-u^{\prime} v_{t}^{\prime}\right) / f$ will be explained in a future study (Aiki et al. 2015, unpublished manuscript).

\section{APPENDIX B}

\section{The Vertical Component of the Vector Streamfunction Associated with the Stokes-Drift Velocity}

For neutral waves, both $\overline{\xi^{\prime} v^{\prime}} \simeq-\overline{\eta^{\prime} u^{\prime}} \simeq\left(\overline{v^{\prime} \xi^{\prime}-u^{\prime} \eta^{\prime}}\right) / 2$ and $\overline{\xi^{\prime} u^{\prime}} \simeq 0 \simeq \overline{\eta^{\prime} v^{\prime}}$ hold. Substitution of these relationships to the approximated expression of the Stokes-drift velocity in (12a) and (12b) yields

$$
\begin{aligned}
& \left\langle\left\langle u^{\text {Stokes }}, v^{\text {Stokes }}, w^{\text {Stokes }}\right\rangle\right\rangle \\
& \left.\simeq-\nabla \times\left\langle\left\langle-\overline{\zeta^{\prime} v^{\prime}}, \overline{\zeta^{\prime} u^{\prime}}, \overline{\left(v^{\prime} \xi^{\prime}-u^{\prime} \eta^{\prime}\right.}\right) / 2\right\rangle\right\rangle,
\end{aligned}
$$

which includes the definition of the vertical component of the Stokes-drift velocity $w^{\text {Stokes }}$. The quantity $\left\langle\left\langle-\overline{\zeta^{\prime} v^{\prime}}, \overline{\zeta^{\prime} u^{\prime}},\left(\overline{v^{\prime} \xi^{\prime}-u^{\prime} \eta^{\prime}}\right) / 2\right\rangle\right\rangle$ is herein referred to as the vector streamfunction associated with the Stokesdrift velocity. Because $\xi^{\prime}$ and $\eta^{\prime}$ are not easily available from model output, it will be useful to rewrite the vertical component of the streamfunction associated with the Stokes-drift velocity in terms of basic quantities, such as $u^{\prime}, v^{\prime}, \zeta^{\prime}, p^{\prime}, q^{\prime}$, and $N^{2}$. We shall suggest two separate expressions. The first expression is given by

$$
\left(\overline{v^{\prime} \xi^{\prime}-u^{\prime} \eta^{\prime}}\right) / 2 \simeq-\overline{u^{\prime} \eta^{\prime}}=\overline{u^{\prime} q^{\prime}} / \beta
$$


where (11), (25c), and (25d) have been used. Because (11) has been used, this expression is applicable to both EQWs and MRWs, but not to MIGWs.
The second expression for the vertical component of the vector streamfunction associated with the Stokes-drift velocity may be obtained by applying a low-pass filter to the virial theorem (35) and then substituting (37) to yield

$$
\begin{aligned}
\left(\overline{v^{\prime} \xi^{\prime}-u^{\prime} \eta^{\prime}}\right) / 2 & \left.=[-\bar{K}+\bar{G}+\bar{\Lambda}] / f+[\underbrace{\overline{\bar{\xi}^{\prime} u^{\prime}+\eta^{\prime} v^{\prime}}}_{\simeq 0}) /(2 f)\right]_{t} \\
& =\underbrace{\left(\overline{-\pi_{y}^{\prime} p_{x}^{\prime}+\pi_{x}^{\prime} p_{y}^{\prime}}\right) /\left(2 f^{2}\right)}_{\simeq 0 \text { for plane waves }}-\bar{K} / f+\left(\overline{-v^{\prime} p_{x}^{\prime}+u^{\prime} p_{y}^{\prime}}\right) /\left(2 f^{2}\right)+[\underbrace{\left[\overline{\bar{\xi}^{\prime} u^{\prime}+\eta^{\prime} v^{\prime}}\right.}_{\simeq 0}) /(2 f)]_{t},
\end{aligned}
$$

where the first line may be compared with the expressions of M06 and TN01. For MIGWs, substitution of $\bar{\Lambda} \simeq 0$ (Table 2) to the first line of (B3) yields $\left(\overline{v^{\prime} \xi^{\prime}-u^{\prime} \eta^{\prime}}\right) / 2 \simeq$ $-\bar{K}+\bar{G}$, which is the expression of M06 that has originally been derived from an analytical solution for the waves. For MRWs, substitution of $\bar{\Lambda} \simeq-\bar{E}$ (Table 2) to the first line of (B3) yields $\left(\overline{v^{\prime} \xi^{\prime}-u^{\prime} \eta^{\prime}}\right) / 2 \simeq 2 \bar{K} / f$, which is consistent with the expression of TN01 [see the third line in their (49)] that has originally been derived from the quasigeostrophic equations.

The second line of (B3) may be compared with the expression of KS13. The sum of the second and third terms on the second line of (B3) corresponds to (2.15) of KS13, which has originally been derived from a combined analytical solution for MIGWs and MRWs. Kinoshita and Sato (2014, hereafter KS14) have shown that (2.15) of $\mathrm{KS} 13$ is not applicable to EQWs, ${ }^{\mathrm{B} 1}$ which we are able to attribute to the first term on the second line of (B3). We note that this term is nonzero for EQWs and automatically vanishes for both MRWs and MIGWs

\footnotetext{
${ }^{\text {B1 }} \mathrm{KS} 14$ did not extend (2.15) of KS13 to EQWs but presented a separate expression for the Stokes-drift velocity associated with zonally propagating EQWs. This is shown in (2.28) of KS14: $-\overline{u^{\prime} \eta^{\prime}} \simeq\left(\overline{v^{\prime} v^{\prime}}-\bar{K}+\bar{G}\right)_{y} / \beta=\left(\bar{E}-\overline{u^{\prime} u^{\prime}}\right)_{y} / \beta$, a result that has been derived from an analytical solution based on the Hermite polynomials (Matsuno 1966). We suggest that a clearer way to rederive their expression is to use the meridional component of the IB pseudomomentum equation (27b) in the present paper and then assume zonally propagating steady waves $\left(\overline{u^{\prime} v^{\prime}} \simeq 0\right.$ has already been explained in the third-to-last paragraph of section $2 \mathrm{~h}$ ). The assumption of vertically homogeneous waves is also necessary to exclude the last term of (27b), which is implicit in the analytical solution used in KS14. It is an open question what motivated them to address their (2.28), because another expression for the streamfunction $-\overline{u^{\prime} \eta^{\prime}} \simeq \overline{u^{\prime} q^{\prime}} / \beta$ in (B2) is easily derived from fewer assumptions and is applicable to all types of EQWs (including meridionally propagating equatorial inertia-gravity waves) as well as MRWs (Table 2).
}

[see discussion after (38)]. Nevertheless, it would be sometimes difficult in the model diagnosis to distinguish whether waves are nearly plane (i.e., MIGWs and MRWs) or nonplane (i.e., EQWs) in the horizontal direction, particularly at low latitudes (Brunet and Haynes 1996). Moreover, the quantity $\pi^{\prime} \equiv \int^{t} p^{\prime} d t$ is not easily available from model output. Hence we shall seek an alternative expression for the first term on the last line of (B3):

$$
\begin{aligned}
\underbrace{\left(\overline{-\pi_{y}^{\prime} p_{x}^{\prime}+\pi_{x}^{\prime} p_{y}^{\prime}}\right) /\left(2 f^{2}\right)}_{\simeq 0 \text { for plane waves }} & =\left(\overline{-\pi_{y}^{\prime} \pi_{t x}^{\prime}+\pi_{x}^{\prime} \pi_{t y}^{\prime}}\right) /\left(2 f^{2}\right) \\
& \simeq\left(\overline{\pi_{y}^{\prime} \pi_{\theta \theta}^{\prime}-\pi_{\theta}^{\prime} \pi_{\theta y}^{\prime}}\right) \sigma k /\left(2 f^{2}\right) \\
& \simeq-\overline{\pi_{y}^{\prime} \pi^{\prime}} \sigma k / f^{2} \simeq \overline{p_{y}^{\prime} \pi_{x}^{\prime}} / f^{2} \\
& =-\overline{p_{y}^{\prime}\left(u^{\prime}-f \eta^{\prime}\right)} / f^{2} \\
& =\underbrace{-\overline{p_{y}^{\prime}\left(u^{\prime} / f+q^{\prime} / \beta\right)} / f}_{\text {Excluded for MIGWs }},
\end{aligned}
$$

where the expression on the last line consists of quantities, such as $u^{\prime}, p^{\prime}$, and $q^{\prime}$, that are easily available from model output. The second line of (B4) has been derived using (25a) and $(25 \mathrm{c})$, the penultimate line has been derived using (16a), and the last line has been derived using (11), which is valid for both MRWs and EQWs $\left(\eta^{\prime}=-q^{\prime} / \beta\right.$ is appropriate), but not for MIGWs $\left(\eta^{\prime}=-q^{\prime} / \beta\right.$ is not appropriate). We suggest distinguishing waves depending on whether $\eta^{\prime} \neq-q^{\prime} / \beta$ (i.e., MIGWs) or $\eta^{\prime}=-q^{\prime} / \beta$ (i.e., MRWs and EQWs) in the model diagnosis. For the type of waves with $\eta^{\prime}=-q^{\prime} / \beta$, either (B2) or the combination of (B3) and (B4) may be used.

To summarize, the two separate expressions (B2) and (B3) are complementary to each other for the understanding of the characteristics of the Stokes-drift 
velocity associated with MIGWs, MRWs, and EQWs (Table 2). See also Constantin (2013) and references therein for literature concerning the Stokes-drift velocity associated with EQWs.

\section{APPENDIX C}

\section{How to Refer to the Quantity $\Lambda$ in a Future Study}

In the present study, the quantity $\Lambda=\left[\left(\xi^{\prime} p^{\prime}\right)_{x}+\right.$ $\left.\left(\eta^{\prime} p^{\prime}\right)_{y}+\left(\zeta^{\prime} p^{\prime}\right)_{z}\right] / 2$ has been referred to as a divergenceform wave-induced pressure. It will be useful to find a more appropriate name for $\Lambda$. The name should indicate for what type of waves the quantity $\bar{\Lambda}$ becomes nonzero. As explained below, $\bar{\Lambda}$ may become nonzero for a type of wave that contains, in any direction, either an "asymmetric velocity" structure or a "trapped modal" structure.

The term "asymmetric velocity" is intended to describe, for example, the meridional structure of the ageostrophic velocity associated with MRWs. The perturbation velocity may be decomposed as $\left\langle\left\langle u^{\prime}, v^{\prime}, w^{\prime}\right\rangle\right\rangle=$ $\left\langle\left\langle-\psi_{y}^{\prime}, \psi_{x}^{\prime}, 0\right\rangle\right\rangle+\left\langle\left\langle u^{a}, v^{a}, w^{a}\right\rangle\right\rangle$, where $\psi^{\prime}=p^{\prime} / f_{0}$ is the geostrophic streamfunction (see footnote 8) and $\left\langle\left\langle u^{a}, v^{a}, w^{a}\right\rangle\right\rangle$ is the ageostrophic component of velocity associated with MRWs. Under the quasigeostrophic approximation, the momentum equations (6a) and (6b) may be rewritten as

$$
\begin{gathered}
-\psi_{y t}^{\prime}-f_{0} v^{a}-\beta y \psi_{x}^{\prime}=0 \quad \text { and } \\
\psi_{x t}^{\prime}+f_{0} u^{a}-\beta y \psi_{y}^{\prime}=0 .
\end{gathered}
$$

These equations indicate that, owing to the presence of the $\beta$ term, the ageostrophic velocity cannot be written in the form of the linear combination of sine and cosine functions in the meridional direction, even if the geostrophic streamfunction is written in the form of nearly plane waves in the horizontal direction. A related discussion appears in AM78b (footnote, p. 654), which may be traced back to Longuet-Higgins (1964). It can be said that nonzero $\bar{\Lambda}$ for MRWs is attributed mostly to the meridional gradient $\left(\overline{\eta^{\prime} p^{\prime}}\right)_{y}$, where $\eta^{\prime}$ includes the effect of the ageostrophic component of velocity $v^{a}$.

The term "trapped modal" is intended to describe, for example, the meridional structure of EQWs. It can be said that nonzero $\bar{\Lambda}$ for EQWs is attributed mostly to $\left(\overline{\eta^{\prime} p^{\prime}}\right)_{y}$. On the other hand, if the buoyancy frequency $N$ varies in the vertical direction, waves may contain a trapped modal structure in the vertical direction, as has been noted for some types of oceanic internal waves in previous studies. For simplicity, let's consider horizontally homogeneous waves (which may be appropriate for MIGWs but not for EQWs and MRWs). Then $\bar{\Lambda} \simeq\left(\overline{\zeta^{\prime} p^{\prime}}\right)_{z} / 2=-\left(\overline{p_{z}^{\prime} p^{\prime}} / N^{2}\right)_{z} / 2$, where (9) has been used. There is a possibility that $\left(\overline{p_{z}^{\prime} p^{\prime}} / N^{2}\right)_{z} \neq 0$ for vertically trapped modal waves, although details of this will be investigated in a future study. This remark is in contrast to the statement " $\bar{\Lambda}=0$ for MIGWs and $\bar{\Lambda}=-\bar{E}$ for MRWs," which frequently appears in the present paper, aimed at highlighting the difference in the direction of the CE and IB fluxes. Careful readers should be aware that the statement " $\bar{\Lambda}=0$ for MIGWs" is actually based on the analytical solution of vertically plane waves, wherein $N$ is assumed to be a vertically uniform constant, as noted in both section $3 \mathrm{c}$ and appendix A.

Taking together the asymmetric-velocity structure and the trapped-modal structure mentioned above, $\Lambda$ might be referred to as, for example, "wavecline pressure" where "cline" indicates gradient in any direction for basic wave structures. Although it is beyond the scope of the present study, we have confirmed that $\bar{\Lambda}$ becomes nonzero for oceanic surface gravity waves (not shown) and is closely related to the depth-dependent expression of the radiation stress as formulated by Aiki and Greatbatch (2013). Indeed, the vertical structure of oceanic surface gravity waves is written (at the leading order in terms of an asymptotic expansion) using an exponential or hyperbolic function and may be regarded as a vertically trapped mode.

An alternative option is to refer to $\Lambda$ as either "waveanisotropy pressure" or "guided-wave pressure," referencing the directional properties of wave propagation in a three-dimensional space.

\section{REFERENCES}

Aiki, H., and T. Yamagata, 2006: Energetics of the layer-thickness form drag based on an integral identity. Ocean Sci., 2, 161-171, doi:10.5194/os-2-161-2006.

_ and K. J. Richards, 2008: Energetics of the global ocean: The role of layer-thickness form drag. J. Phys. Oceanogr., 38, 18451869, doi:10.1175/2008JPO3820.1.

, and R. J. Greatbatch, 2013: The vertical structure of the surface wave radiation stress for circulation over a sloping bottom as given by thickness-weighted-mean theory. J. Phys. Oceanogr., 43, 149-164, doi:10.1175/JPO-D-12-059.1.

$\longrightarrow$, and - 2014: A new expression for the form stress term in the vertically Lagrangian mean framework for the effect of surface waves on the upper-ocean circulation. J. Phys. Oceanogr., 44, 3-23, doi:10.1175/JPO-D-12-0228.1.

Andrews, D. G., 1983a: A finite-amplitude Eliassen-Palm theorem in isentropic coordinates. J. Atmos. Sci., 40, 1877-1883, doi:10.1175/1520-0469(1983)040<1877:AFAEPT>2.0.CO;2. 1983b: A conservation law for small-amplitude quasigeostrophic disturbances on a zonally asymmetric basic flow. J. Atmos. Sci., 40, 85-90, doi:10.1175/1520-0469(1983)040<0085: ACLFSA $>2.0 . \mathrm{CO} ; 2$. 
_ 1984: On the existence of nonzonal flows satisfying sufficient conditions for instability. Geophys. Astrophys. Fluid Dyn., 28, 243-256, doi:10.1080/03091928408230366.

_ , and M. E. McIntyre, 1976: Planetary waves in horizontal and vertical shear: The generalized Eliassen-Palm relation and the mean zonal acceleration. J. Atmos. Sci., 33, 2031-2048, doi:10.1175/1520-0469(1976)033<2031:PWIHAV>2.0.CO;2.

— a Lagrangian-mean flow. J. Fluid Mech., 89, 609-646, doi:10.1017/S0022112078002773.

— Mech., 89, 647-664, doi:10.1017/S0022112078002785.

Ardhuin, F., N. Rascle, and K. Belibassakis, 2008: Explicit wave-averaged primitive equations using a generalized Lagrangian mean. Ocean Modell., 20, 35-60, doi:10.1016/ j.ocemod.2007.07.001.

Bleck, R., 1985: On the conversion between mean and eddy components of potential and kinetic energy in isentropic and isopycnic coordinates. Dyn. Atmos. Oceans, 9, 17-37, doi:10.1016/0377-0265(85)90014-4.

Bretherton, F. P., 1966: Critical layer instability in baroclinic flows. Quart. J. Roy. Meteor. Soc., 92, 325-334, doi:10.1002/ qj. 49709239302.

, and C. J. R. Garrett, 1968: Wavetrains in inhomogeneous moving media. Proc. Roy. Soc. London, A302, 529-554, doi:10.1098/rspa.1968.0034.

Brunet, G., and P. H. Haynes, 1996: Low-latitude reflection of Rossby wave trains. J. Atmos. Sci., 53, 482-496, doi:10.1175/ 1520-0469(1996)053<0482:LLRORW >2.0.CO;2.

Bühler, O., 2009: Waves and Mean Flows. Cambridge University Press, $341 \mathrm{pp}$.

, 2014: A gentle stroll through EP flux theory. Eur. J. Mech., 47B, 12-15, doi:10.1016/j.euromechflu.2014.01.010.

Cai, M., and B. Huang, 2013: A dissection of energetics of the geostrophic flow: Reconciliation of Rossby wave energy flux and group velocity. J. Atmos. Sci., 70, 2179-2196, doi:10.1175/ JAS-D-12-0249.1.

Chang, E. K. M., and I. Orlanski, 1994: On energy flux and group velocity of waves in baroclinic flows. J. Atmos. Sci., 51, 3823-3828, doi:10.1175/1520-0469(1994)051<3823:OEFAGV>2.0.CO;2.

Charney, J., and P. Drazin, 1961: Propagation of planetary-scale disturbances from the lower into the upper atmosphere. J. Geophys. Res., 66, 83-109, doi:10.1029/JZ066i001p00083.

Chu, V. H., and C. C. Mei, 1970: On slowly-varying Stokes waves. J. Fluid Mech., 41, 873-887, doi:10.1017/S0022112070000988.

Constantin, A., 2013: Some three-dimensional nonlinear equatorial flows. J. Phys. Oceanogr., 43, 165-175, doi:10.1175/JPO-D-12-062.1.

Craik, A. D. D., and S. Leibovich, 1976: A rational model for Langmuir circulations. J. Fluid Mech., 73, 401-426, doi:10.1017/S0022112076001420.

Dritschel, D. G., and M. E. McIntyre, 2008: Multiple jets as PV staircases: The Phillips effect and the resilience of eddy-transport barriers. J. Atmos. Sci., 65, 855-873, doi:10.1175/2007JAS2227.1.

Durran, D. R., 1988: On a physical mechanism for Rossby wave propagation. J. Atmos. Sci., 45, 4020-4022, doi:10.1175/ 1520-0469(1988)045<4020:OAPMFR > 2.0.CO;2.

Eckart, C., 1963: Extension of Howard's circle theorem to adiabatic jets. Phys. Fluids, 6, 1042-1047, doi:10.1063/1.1706860.

Eden, C., R. J. Greatbatch, and J. Willebrand, 2007: A diagnosis of thickness fluxes in an eddy-resolving model. J. Phys. Oceanogr., 37, 727-742, doi:10.1175/JPO2987.1.

Eliassen, A., and E. Palm, 1960: On the transfer of energy in stationary mountain waves. Geofys. Publ., 22, 1-23.
Gent, P. R., J. Willebrand, T. J. McDougall, and J. C. McWilliams, 1995: Parameterizing eddy-induced tracer transports in ocean circulation models. J. Phys. Oceanogr., 25, 463-474, doi:10.1175/1520-0485(1995)025<0463:PEITTI >2.0.CO;2.

Gill, A. E., 1982: Atmosphere-Ocean Dynamics. International Geophysics Series, Vol. 30, Academic Press, 662 pp.

Greatbatch, R. J., 1998: Exploring the relationship between eddy-induced transport velocity, vertical momentum transfer, and the isopycnal flux of potential vorticity. J. Phys. Oceanogr., 28, 422-432, doi:10.1175/1520-0485(1998)028<0422: ETRBEI $>2.0 . \mathrm{CO} ; 2$.

_ _ 2001: A framework for mesoscale eddy parameterization based on density-weighted averaging at fixed height. J. Phys. Oceanogr., 31, 2797-2806, doi:10.1175/1520-0485(2001)031<2797: AFFMEP $>2.0 . \mathrm{CO} ; 2$.

— , and T. J. McDougall, 2003: The non-Boussinesq temporal residual mean. J. Phys. Oceanogr., 33, 1231-1239, doi:10.1175/ 1520-0485(2003)033<1231:TNTRM > 2.0.CO;2.

Hasselmann, K., 1971: On the mass and momentum transfer between short gravity waves and larger-scale motions. J. Fluid Mech., 50, 189-205, doi:10.1017/S0022112071002520.

Hayashi, Y.-Y., and W. R. Young, 1987: Stable and unstable shear modes of rotating parallel flows in shallow water. J. Fluid Mech., 184, 477-504, doi:10.1017/S0022112087002982.

Haynes, P. H., 1988: Forced, dissipative generalizations of finiteamplitude wave-activity conservation relations for zonal and nonzonal basic flows. J. Atmos. Sci., 45, 2352-2362, doi:10.1175/1520-0469(1988)045<2352:FDGOFA > 2.0.CO;2.

Held, I. M., 1985: Pseudomomentum and the orthogonality of modes in shear flows. J. Atmos. Sci., 42, 2280-2288, doi:10.1175/1520-0469(1985)042<2280:PATOOM>2.0.CO;2.

Holliday, D., and M. E. McIntyre, 1981: On potential energy density in an incompressible, stratified fluid. J. Fluid Mech., 107, 221-225, doi:10.1017/S0022112081001742.

Holton, J. R., 1992: An Introduction to Dynamic Meteorology. 3rd ed. Academic Press, 511 pp.

Horinouchi, T., and S. Yoden, 1998: Wave-mean flow interaction associated with a QBO-like oscillation simulated in a simplified GCM. J. Atmos. Sci., 55, 502-526, doi:10.1175/ 1520-0469(1998)055<0502:WMFIAW $>2.0 . \mathrm{CO} ; 2$.

Hoskins, B. J., I. N. James, and G. H. White, 1983: The shape, propagation and mean-flow interaction of large-scale weather systems. J. Atmos. Sci., 40, 1595-1612, doi:10.1175/ 1520-0469(1983)040<1595:TSPAMF $>2.0 . \mathrm{CO} ; 2$.

Ishioka, K., and S. Yoden, 1996: Numerical methods of estimating bounds on the non-linear saturation of barotropic instability. J. Meteor. Soc. Japan, 74, 167-174.

Iwasaki, T., 1989: A diagnostic formulation for wave-mean flow interactions and Lagragian-mean circulation with a hybrid vertical coordinate of pressure and isentropes. J. Meteor. Soc. Japan, 67, 293-312.

_- 2001: Atmospheric energy cycle viewed from wave-meanflow interaction and Lagrangian mean circulation. J. Atmos. Sci., 58, 3036-3052, doi:10.1175/1520-0469(2001)058<3036: AECVFW $>2.0 . \mathrm{CO} ; 2$.

Jacobson, T., and H. Aiki, 2006: An exact energy for TRM theory. J. Phys. Oceanogr., 36, 558-564, doi:10.1175/JPO2872.1.

Kinoshita, T., and K. Sato, 2013: A formulation of threedimensional residual mean flow applicable both to inertiagravity waves and to Rossby waves. J. Atmos. Sci., 70, 1577-1602, doi:10.1175/JAS-D-12-0137.1.

, and _ 2014: A formulation of three-dimensional residual mean flow and wave activity flux applicable to equatorial 
waves. J. Atmos. Sci., 71, 3427-3438, doi:10.1175 JAS-D-13-0161.1.

Lagrange, J. L., 1788: Méchanique Analitique. Chez la Veuve Desaint, $512 \mathrm{pp}$.

Longuet-Higgins, M. S., 1953: Mass transport in water waves. Philos. Trans. Roy. Soc. London, A245, 535-581, doi:10.1098/rsta.1953.0006.

_ 1964: On group velocity and energy flux in planetary wave motions. Deep-Sea Res. Oceanogr. Abstr., 11, 35-42, doi:10.1016/0011-7471(64)91080-0.

_ 1969: On the transport of mass by time-varying ocean currents. Deep-Sea Res. Abstr., 16, 431-447, doi:10.1016/ 0011-7471(69)90031-X.

Maddison, J. R., and D. P. Marshall, 2013: The Eliassen-Palm flux tensor. J. Fluid Mech., 729, 69-102, doi:10.1017/jfm.2013.259.

Masuda, A., 1978: Group velocity and energy transport by Rossby waves. J. Oceanogr. Soc. Japan, 34, 1-7, doi:10.1007/BF02109610.

Matsuno, T., 1966: Quasi-geostrophic motions in the equatorial area. J. Meteor. Soc. Japan, 44, 25-43.

McDougall, T. J., and P. C. McIntosh, 2001: The temporal-residualmean velocity. Part II: Isopycnal interpretation and the tracer and momentum equations. J. Phys. Oceanogr., 31, 1222-1246, doi:10.1175/1520-0485(2001)031<1222:TTRMVP>2.0.CO;2.

McPhaden, M. J., and P. Ripa, 1990: Wave-mean flow interactions in the equatorial ocean. Annu. Rev. Fluid Mech., 22, 167-205, doi:10.1146/annurev.fl.22.010190.001123.

Methven, J., 2013: Wave activity for large-amplitude disturbances described by the primitive equations on the sphere. J. Atmos. Sci., 70, 1616-1630, doi:10.1175/JAS-D-12-0228.1.

Miyahara, S., 2006: A three dimensional wave activity flux applicable to inertio-gravity waves. SOLA, 2, 108-111, doi:10.2151/ sola.2006-028.

Müller, P., 1995: Ertel's potential vorticity theorem in physical oceanography. Rev. Geophys., 33, 67-97, doi:10.1029/94RG03215.

Nakamura, N., and A. Solomon, 2011: Finite-amplitude wave activity and mean flow adjustments in the atmospheric general circulation. Part II: Analysis in the isentropic coordinate. J. Atmos. Sci., 68, 2783-2799, doi:10.1175/2011JAS3685.1.

Noda, A., 2010: A general three-dimensional transformed Eulerain mean formulation. SOLA, 6, 85-88, doi:10.2151/sola.2010-022

Plumb, R. A., 1985: On the three-dimensional propagation of stationary waves. J. Atmos. Sci., 42, 217-229, doi:10.1175/ 1520-0469(1985)042<0217:OTTDPO > 2.0.CO;2.

- 1986: Three-dimensional propagation of transient quasigeostrophic eddies and its relationship with the eddy forcing of the time-mean flow. J. Atmos. Sci., 43, 1657-1678, doi:10.1175/1520-0469(1986)043<1657:TDPOTQ > 2.0.CO;2.

Rhines, P., 1982: Basic dynamics of the large-scale geostrophic circulation. 1982 summer study program in geophysical fluid dynamics, Woods Hole Oceanographic Institution Tech Rep. WHOI-82-45, 1-4, doi:10.1575/1912/2895.
Ripa, P., 1982: Nonlinear wave-wave interactions in a one-layer reduced-gravity model on the equatorial $\beta$ plane. J. Phys. Oceanogr., 12, 97-111, doi:10.1175/1520-0485(1982)012<0097: NWIIAO $>2.0 . \mathrm{CO} ; 2$

Sassi, F., and R. R. Garcia, 1997: The role of equatorial waves forced by convection in the tropical semiannual oscillation. J. Atmos. Sci., 54, 1925-1942, doi:10.1175/1520-0469(1997)054<1925: TROEWF $>2.0 . \mathrm{CO} ; 2$

Sato, K., and T. J. Dunkerton, 1997: Estimates of momentum flux associated with equatorial Kelvin and gravity waves. J. Geophys. Res., 102, 26 247-26261, doi:10.1029/96JD02514.

Scinocca, J. F., and T. G. Shepherd, 1992: Nonlinear wave-activity conservation laws and Hamiltonian structure for the twodimensional anelastic equations. J. Atmos. Sci., 49, 5-28, doi:10.1175/1520-0469(1992)049<0005:NWACLA >2.0.CO;2.

Solomon, A., and N. Nakamura, 2012: An exact Lagrangian-mean wave activity for finite-amplitude disturbances to barotropic flow on a sphere. J. Fluid Mech., 693, 69-92, doi:10.1017/jfm.2011.460.

Takaya, K., and H. Nakamura, 1997: A formulation of a waveactivity flux for stationary Rossby waves on a zonally varying basic flow. Geophys. Res. Lett., 24, 2985-2988, doi:10.1029/ 97GL03094.

- , and - 2001: A formulation of a phase-independent wave-activity flux for stationary and migratory quasigeostrophic eddies on a zonally varying basic flow. J. Atmos. Sci., 58, 608-627, doi:10.1175/1520-0469(2001)058<0608: AFOAPI $>2.0 . \mathrm{CO} ; 2$.

Takehiro, S.-I., and Y.-Y. Hayashi, 1992: Over-reflection and shear instability in a shallow-water model. J. Fluid Mech., 236, 259_ 279, doi:10.1017/S0022112092001411.

Taylor, G. I., 1915: Eddy motion in the atmosphere. Philos. Trans. Roy. Soc. London, A215, 1-23, doi:10.1098/rsta.1915.0001.

Trenberth, K. E., 1986: An assessment of the impact of transient eddies on the zonal flow during a blocking episode using localized Eliassen-Palm flux diagnostics. J. Atmos. Sci., 43, 2070-2087, doi:10.1175/1520-0469(1986)043<2070: AAOTIO $>2.0 . \mathrm{CO} ; 2$.

Tung, K. K., 1986: Nongeostrophic theory of zonally averaged circulation. Part I: Formulation. J. Atmos. Sci., 43, 2600-2618, doi:10.1175/1520-0469(1986)043<2600:NTOZAC >2.0.CO;2.

Uryu, M., 1974: Mean zonal flows induced by a vertically propagating Rossby wave packet. J. Meteor. Soc. Japan, 52, 481-490.

Whitham, G. B., 1974: Linear and Nonlinear Waves. John Wiley \& Sons, Inc., 636 pp.

Yanai, M., and T. Maruyama, 1966: Stratospheric wave disturbances propagating over the equatorial Pacific. J. Meteor. Soc. Japan, 44, 291-294.

Young, W. R., 2012: An exact thickness-weighted average formulation of the Boussinesq equations. J. Phys. Oceanogr., 42, 692-707, doi:10.1175/JPO-D-11-0102.1. 